\title{
Notes on GIT and symplectic reduction for bundles and varieties
}

\author{
R.P. Thomas
}

\section{Introduction}

These notes give an introduction to Geometric Invariant Theory (GIT) and symplectic reduction, with lots of pictures and simple examples. We describe their applications to moduli of bundles and varieties, leaving the technical work on the analysis of the Hilbert-Mumford criterion in these situations to the final sections. We outline their infinite dimensional analogues (so called Hitchin-Kobayashi correspondences) in gauge theory and in the theory of constant scalar curvature Kähler (cscK) and Kähler-Einstein (KE) metrics on algebraic varieties. Donaldson's work on why these should be thought of as the classical limits of the original finite dimensional constructions - which are then their "quantisations" - is explained. The many analogies and connections between the bundle and variety cases are emphasised; in particular the GIT analysis of stability of bundles is shown to be a special case of the (harder) problem for varieties.

For GIT we work entirely over $\mathbb{C}$ and skip or only sketch many proofs. The interested reader should refer to the excellent [Dl, GIT, Ne] for more details. Throughout this survey we mention many names, but only include certain key papers in the references - apologies to those omitted but compiling a truly comprehensive bibliography would be fraught with danger.

Acknowledgements. I have learnt GIT from Simon Donaldson and Frances Kirwan over many years. Large parts of these notes are nothing but an account of Donaldson's point of view on GIT and symplectic reduction for moduli of varieties. The last sections describe joint work with Julius Ross, and I would like to thank him, Gábor Székelyhidi and Xiaowei Wang for useful conversations. Thanks also to Claudio Arezzo, Joel Fine, Daniel Huybrechts, Julien Keller, Dmitri Panov, Michael Singer and Burt Totaro for comments on the manuscript. 


\section{A brief review of affine and projective geometry}

This section can be safely skipped by readers with any knowledge of algebraic geometry. We fix some notation and speedily review some standard theory of complex affine and projective varieties (and schemes). These are much simpler than arbitrary varieties in that they can be described by a single ring. Throughout "ring" means finitely generated commutative $\mathbb{C}$ algebra - i.e. a Noetherian ring with a scalar action of $\mathbb{C}$ making it into a $\mathbb{C}$-vector space and commuting with multiplication.

Affine varieties. Affine varieties $X$ are just the irreducible components of the zero sets of finite collections of polynomials $p_{1}, \ldots, p_{k}$ in some affine space $\mathbb{C}^{n}$. They are in one-to-one correspondence with rings with no zero divisors (i.e., integral domains); in coordinates this is particularly simple:

$$
\begin{aligned}
& \mathbb{C}\left[x_{1}, \ldots, x_{n}\right] \longleftrightarrow \mathbb{C}^{n}, \\
& \frac{\mathbb{C}\left[x_{1}, \ldots, x_{n}\right]}{\left(p_{1}, \ldots, p_{k}\right)} \longleftrightarrow\left(p_{1}=0=\ldots=p_{k}\right) \subseteq \mathbb{C}^{n}, \\
& \mathcal{O}_{X} \quad \longleftrightarrow \quad X .
\end{aligned}
$$

The arrow $\leftarrow$ replaces a variety by the ring of functions on it (i.e. the functions $\mathbb{C}\left[x_{1}, \ldots, x_{n}\right]$ on $\mathbb{C}^{n}$ divided out by the ideal of those that vanish on $X$ ). The arrow $\rightarrow$ recovers $X$ from its ring of functions $\mathcal{O}_{X}$ by taking a finite number of generators $x_{1}, \ldots, x_{n}$ and a finite (by the Hilbert basis theorem) number of relations $p_{1}, \ldots, p_{k}$ (considered as polynomials in the generators) and setting $X$ to be the affine variety in $\mathbb{C}^{n}$ cut out by the polynomials $p_{i}$.

The embedding is equivalent to the choice of generators: each is a map $X \rightarrow \mathbb{C}$ so $n$ of them give the map $X \hookrightarrow \mathbb{C}^{n}$. (Invariantly, we embed $X$ in the dual of the vector space on the generators,

$$
X \ni x \mapsto e v_{x} \in\left(\mathbb{C}\left\langle x_{1}, \ldots, x_{n}\right\rangle\right)^{*}, \quad e v_{x}(f):=f(x),
$$

each point $x$ of $X$ mapping to the linear functional that evaluates functions at $x$.) The ideal of functions $\left(p_{1}, \ldots, p_{k}\right)$ vanishing on $X \subset \mathbb{C}^{n}$ is prime (if it contains $f g$ then it contains one of $f$ or $g$ ) reflecting the fact that the ring $\mathcal{O}_{X}$ has no zero divisors and that $X$ is irreducible.

So really $(2.1)$ is a correspondence between

(1) Affine varieties $X \subseteq \mathbb{C}^{n}$ with a fixed embedding into $n$-dimensional affine space;

(2) prime ideals $I \subseteq \mathbb{C}\left[x_{1}, \ldots, x_{n}\right]$, and

(3) rings without zero divisors plus a choice of $n$ generators.

The coordinate-free approach (which also shows the above construction is independent of choices) is to note that the points $x$ of $X$ are in one-to-one correspondence with the maximal ideals $\mathscr{I}_{x} \subset \mathcal{O}_{X}$ of functions vanishing at $x$. So to any ring without zero divisors $R$ we associate an affine variety 
Spec $R$ whose points are maximal ideals in $R$; the coordinate-independent version of (2.1):

$$
\mathcal{O}_{X}=R \quad \longleftrightarrow \quad X=\operatorname{Spec} R
$$

This can be extended to a correspondence between arbitrary rings and affine schemes, which are allowed nilpotents in their ring of functions, corresponding to multiplicities or infinitesimal directions in the scheme.

Projective varieties. Now we just do everything $\mathbb{C}^{*}$-equivariantly. Recall that a $\mathbb{C}^{*}$-action on a vector space $V$ is equivalent to a grading, i.e., a splitting into subspaces (the eigen- or weight spaces) $V_{k}$ parameterised by the integers (the eigenvalues or weights). So we replace rings by graded rings, ideals by homogeneous ideals (those which are the sum of their graded pieces - i.e. which are $\mathbb{C}^{*}$-invariant), and get a correspondence between graded rings without zero divisors and projective varieties. This is easiest to express in coordinates when the ring is generated by its degree one piece:

$$
\begin{aligned}
& \mathbb{C}\left[x_{0}, \ldots, x_{n}\right] \quad \longleftrightarrow \quad \mathbb{P}^{n}, \\
& \frac{\mathbb{C}\left[x_{0}, \ldots, x_{n}\right]}{\left(p_{1}, \ldots, p_{k}\right)} \longleftrightarrow\left(p_{1}=0=\ldots=p_{k}\right) \subseteq \mathbb{P}^{n} .
\end{aligned}
$$

Here $\mathbb{C}\left[x_{0}, \ldots, x_{n}\right]$ is given the standard grading it inherits from the scalar $\mathbb{C}^{*}$-action on $\mathbb{C}^{n+1}$ (i.e. the $x_{i}$ have weight one), and the $p_{i}$ are homogeneous polynomials (eigenvectors for the $\mathbb{C}^{*}$-action). They cut out an affine variety $\widetilde{X}=\left(p_{1}=0=\ldots=p_{k}\right)$ in $\mathbb{C}^{n+1}$, which is $\mathbb{C}^{*}$-invariant and so a cone, determined by its set of lines through the origin $X \subseteq \mathbb{P}^{n}$. ( $X$ and $\mathbb{P}^{n}$ are of course the quotients of $\widetilde{X} \backslash\{0\}$ and $\mathbb{C}^{n+1} \backslash\{0\}$ by $\mathbb{C}^{*}$, but we have yet to develop a theory of quotients (!).)

This describes the arrow $\rightarrow$. For $\leftarrow$ we cannot simply take the ring of functions on $X$ since this consists of just the constants; we have to take the ring of functions on the cone $\widetilde{X}$, which can be described on $X$ in terms of a line bundle.

Since $X$ is the space of lines in $\widetilde{X}$, it has a tautological line bundle $\mathcal{O}_{X}(-1)=\left.\mathcal{O}_{\mathbb{P}^{n}}(-1)\right|_{X}$ over it whose fibre over a point in $X$ is the corresponding line in $\widetilde{X} \subseteq \mathbb{C}^{n+1}$. The total space of $\mathcal{O}_{X}(-1)$ therefore has a tautological map to $\widetilde{\widetilde{X}}$ which is an isomorphism away from the zero section $X \subset \mathcal{O}_{X}(-1)$, which is all contracted down to the origin in $\tilde{X}$. In fact the total space of $\mathcal{O}_{X}(-1)$ is the blow up of $\widetilde{X}$ in the origin. 


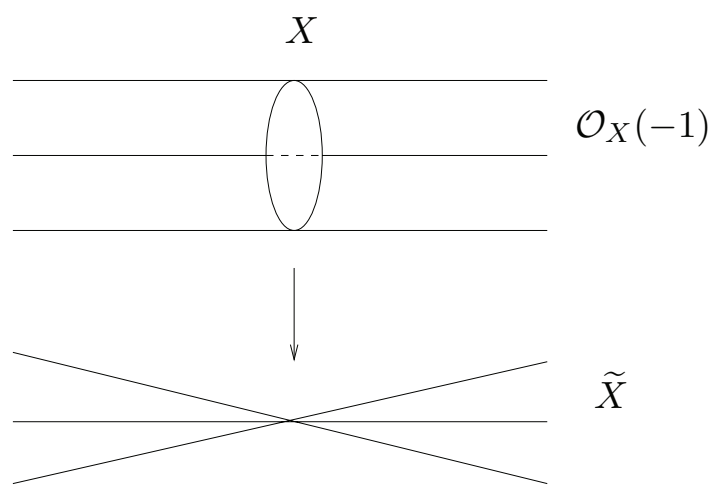

Linear functions on $\mathbb{C}^{n+1}$ like $x_{i}$, restricted to $\widetilde{X}$ and pulled back to the total space of $\mathcal{O}_{X}(-1)$, give functions which are linear on the fibres, so correspond to sections of the dual line bundle $\mathcal{O}_{X}(1)$. Similarly degree $k$ homogeneous polynomials on $\widetilde{X}$ define functions on the total space of $\mathcal{O}_{X}(-1)$ which are of degree $k$ on the fibres, and so give sections of the $k$ th tensor power $\mathcal{O}_{X}(k)$ of the dual of the line bundle $\mathcal{O}_{X}(-1)$.

So the grading that splits the functions on $\widetilde{X}$ into homogeneous degree (or $\mathbb{C}^{*}$-weight spaces) corresponds to sections of different line bundles $\mathcal{O}_{X}(k)$ on $X$. So $\leftarrow$ takes the direct sum $\bigoplus_{k \geq 0} H^{0}\left(\mathcal{O}_{X}(k)\right)$, considered a graded ring by tensoring sections $\mathcal{O}(k) \otimes \mathcal{O}(l) \stackrel{\simeq}{\longrightarrow} \mathcal{O}(k+l)$. For the line bundle $\mathcal{O}_{X}(1)$ sufficiently positive, this ring will be generated in degree one. It is often called the (homogeneous) coordinate ring of the polarised (= endowed with an ample line bundle) variety $\left(X, \mathcal{O}_{X}(1)\right)$.

The degree one restriction is for convenience and can be dropped (by working with varieties in weighted projective spaces), or bypassed by replacing $\mathcal{O}_{X}(1)$ by $\mathcal{O}_{X}(p)$, i.e. using the ring $R^{(p)}=\bigoplus_{k \geq 0} R_{k p}$; for $p \gg 0$ this will be generated by its degree one piece $R_{p}$.

The choice of generators of the ring is what gives the embedding in projective space. In fact the sections of any line bundle $L$ over $X$ define a (rational) map

$$
X \rightarrow \mathbb{P}\left(H^{0}(X, L)^{*}\right), \quad x \mapsto e v_{x}, \quad e v_{x}(s):=s(x),
$$

(compare (2.2)) which in coordinates maps $x$ to $\left(s_{0}(x): \ldots: s_{n}(x)\right) \in \mathbb{P}^{n}$, where $s_{i}$ form a basis for $H^{0}(L)$. This map is only defined for those $x$ with $e v_{x} \neq 0$, i.e., for which $s(x)$ is not zero for every $s$.

It remains to describe $\rightarrow$ in a coordinate-free manner, by noting that the points of $X$, i.e. lines of $\widetilde{X}$, are $\mathbb{C}^{*}$-invariant subvarieties that are minimal among those which are not the origin in $\widetilde{X} \subseteq \mathbb{C}^{n+1}$; i.e. homogeneous ideals of the homogeneous coordinate ring that are maximal amongst all homogeneous ideals minus the one corresponding to the origin. So to any graded ring $R$ we associate a projective variety $\operatorname{Proj} R$ whose points are the homogeneous ideals of $R$ maximal amongst those except the irrelevant ideal $R^{+}:=\bigoplus_{k>0} R_{k}$. It comes equipped with an ample line bundle, the sections 
of whose $k$ th power gives $R_{k}$ for $k \gg 0$. This gives the coordinate-free (not quite one-to-one) correspondence (2.3):

$$
\mathcal{O}_{\tilde{X}}=R \quad \longleftrightarrow \quad\left(X, \mathcal{O}_{X}(1)\right)=\operatorname{Proj} R
$$

Replacing $R$ by $R^{(p)}$ leaves the variety Proj $R$ unaltered but changes the line bundle from $\mathcal{O}_{X}(1)$ to $\mathcal{O}_{X}(p)$.

Similarly arbitrary graded rings with zero divisors (finitely generated, as usual) correspond (not quite one-to-one) to polarised schemes.

Notation. Throughout $G$ will be a connected reductive complex linear algebraic group with Lie algebra $\mathfrak{g}$. Reductive means that all (complex) representations split into sums of irreducibles, but equivalently it is the complexification of a compact real Lie group $K<G$ with Lie algebra $\mathfrak{k}<\mathfrak{g}$ such that $\mathfrak{g}=\mathfrak{k}+i \mathfrak{k}$. Therefore representations are also representations of the compact group, which preserve a hermitian inner product (by averaging any inner product on the representation using Haar measure on $K$ ) and so split into direct sums of irreducibles by taking orthogonal complements to invariant subspaces. These splittings are by complex subspaces, so are then also preserved by the complexification $G$.

For the purposes of these notes one can always assume that $K<G$ is $S^{1}<\mathbb{C}^{*},\left(S^{1}\right)^{m}<\left(\mathbb{C}^{*}\right)^{m}$ or $S U(m)<S L(m, \mathbb{C})$.

\section{Geometric Invariant Theory}

GIT is a way of taking quotients in algebraic geometry. This may sound like a dry and technical subject, but it is beautifully geometric (as we hope to show) and leads, through its link with symplectic reduction, to unexpected mathematics (some of which we describe later).

Suppose we are in the following situation, of $G$ acting on a projective variety $X$ through $S L$ transformations of the projective space.

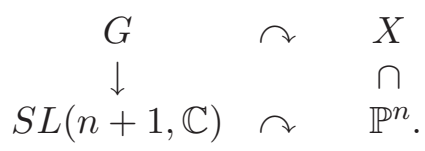

We would like to form a quotient $X / G$, ideally within the same category of projective varieties. There are a number of problems with this.

The topological quotient is not Hausdorff. Since $X$ is compact but $G$ is noncompact, a nontrivial $G$-action cannot be proper. There are nonclosed orbits (with lower dimensional orbits in their closures) so the topological quotient is not Hausdorff. 


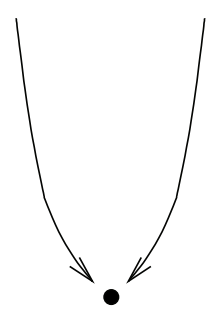

It is clear from the above simple illustration of 3 orbits, all of whose closures contain the smaller orbit, that we must remove some orbits to get a separated (Hausdorff) quotient. In the above case this would be the lower dimensional orbit; just as we would expect to remove the origin from $\mathbb{C}^{n+1}$ if we wanted to quotient by the scalar action of $\mathbb{C}^{*}$ to get $\mathbb{P}^{n}$ (an example to which we shall return).

Removing smaller orbits does not suffice. Another simple example shows that the quotient can still be nonseparated if we remove all lower dimensional orbits. Consider the action of $\mathbb{C}^{*}$ on $\mathbb{C}^{2}$ (or its projective completion $\mathbb{P}^{2} \supset \mathbb{C}^{2}$ ) by matrices

$$
\mathbb{C}^{*} \ni \lambda \mapsto\left(\begin{array}{cc}
\lambda & 0 \\
0 & \lambda^{-1}
\end{array}\right) \in S L(2, \mathbb{C})
$$

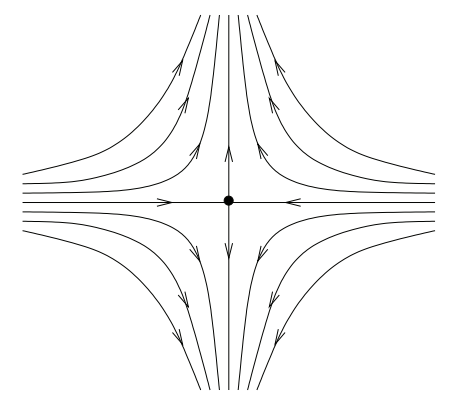

In this case removing the origin would make the topological quotient the affine line with nonseparated doubled point at the origin: it is clear that the punctured (origin removed) $x$ - and $y$-axes are both orbits in the limit of the orbits $\{x y=\alpha\}$ as $\alpha \rightarrow 0$.

In this simple case it is clear that we would like the quotient to be $\mathbb{C}$, with $\alpha \in \mathbb{C} \backslash\{0\}$ representing the orbit $\{x y=\alpha\}$. But what then should the point $\alpha=0$ of this "quotient" represent? There are three standard solutions to the problem.

- Kapranov's Chow quotient (and the closely related Hilbert quotient, of which it is a contraction) parameterises the invariant conics in this example. For $\alpha \neq 0$ these are just the closed orbits of top dimension, while $\alpha=0$ represents the invariant conic $\{x y=0\}$, the union of all 3 bad orbits. We will not say more about the Chow quotient; instead see $[\mathbf{H u}]$, for example (which, unsurprisingly, uses the same example for illustration).

- The Geometric Invariant Theory quotient gives the same for $\alpha \neq 0$, but $\alpha=0$ represents any one of the 3 orbits: GIT identifies all 3 orbits with each other in an equivalence class. 
- The Symplectic reduction throws away the two nonclosed orbits - the (punctured) $x$ - and $y$-axes - and keeps the origin. So in this case the quotient represents the closed orbits, including the lower dimensional one.

In this simple case all three quotients give the same answer $\mathbb{C}$, but in general one expects the Chow quotient to dominate the GIT and symplectic quotients, which are isomorphic.

In general GIT and the symplectic quotient choose certain "unstable" orbits to remove to give a separated quotient. GIT also identifies some "semistable" orbits (those whose closures intersect each other) to compactify the quotient, resulting in a projective variety which we call $X / G$. The symplectic quotient compactifies by taking a distinguished representative of each semistable equivalence class above - the intersection of their closures, which turns out to be the unique closed semistable orbit in the equivalence class.

The construction of the GIT quotient. This is trivial, a formality. We consider $X$ projective first, since although the affine case is often even easier, sometimes it is best embedded in the projective case, as we shall see below.

Since we have assumed that $G$ acts through $S L(n+1, \mathbb{C}$ ) (rather than just its quotient $P S L(n+1, \mathbb{C})$ ), the action lifts from $X$ to one covering it on $\mathcal{O}_{X}(-1)$. In other words we don't just act on the projective space (and $X$ therein) but on the vector space overlying it (and the cone $\widetilde{X}$ on $X$ therein). This is called a linearisation of the action. Thus $G$ acts on each $H^{0}\left(\mathcal{O}_{X}(r)\right)$.

Then, just as $\left(X, \mathcal{O}_{X}(1)\right)$ is determined by its graded ring of sections of $\mathcal{O}(r)$ (i.e., the ring of functions on $\tilde{X})$,

$$
(X, \mathcal{O}(1)) \longleftrightarrow \bigoplus_{r} H^{0}(X, \mathcal{O}(r)),
$$

we simply construct $X / G$ (with a line bundle on it) from the ring of invariant sections:

$$
X / G \longleftrightarrow \bigoplus_{r} H^{0}(X, \mathcal{O}(r))^{G} .
$$

This is sensible, since if there is a good quotient then functions on it pullback to give $G$-invariant functions on $X$, i.e. functions constant on the orbits, the fibres of $X \rightarrow X / G$. For it to work we need

Lemma 3.3. $\bigoplus_{r} H^{0}(X, \mathcal{O}(r))^{G}$ is finitely generated.

Proof. Since $R:=\bigoplus_{r} H^{0}(X, \mathcal{O}(r))$ is Noetherian, Hilbert's basis theorem tells us that the ideal $R .\left(\bigoplus_{r>0} H^{0}(X, \mathcal{O}(r))^{G}\right)$ generated by $R_{+}^{G}:=$ $\bigoplus_{r>0} H^{0}(X, \mathcal{O}(r))^{G}$ is generated by a finite number of elements $s_{0}, \ldots, s_{k} \in$ $R_{+}^{G}$. 
Thus any element $s \in H^{0}(X, \mathcal{O}(r))^{G}, r>0$, may be written $s=$ $\sum_{i=0}^{k} f_{i} s_{i}$, for some $f_{i} \in R$ of degree $<r$. To show that the $s_{i}$ generate $R_{+}^{G}$ as an algebra we must show that the $f_{i}$ can be taken to lie in $R^{G}$.

We now use the fact that $G$ is the complexification of the compact group $K$. Since $K$ has an invariant metric, we can average over it and use the facts that $s$ and $s_{i}$ are invariant to give

$$
s=\sum_{i=0}^{k} A v\left(f_{i}\right) s_{i},
$$

where $A v\left(f_{i}\right)$ is the (K-invariant) $K$-average of $f_{i}$. By complex linearity $A v\left(f_{i}\right)$ is also $G$-invariant (for instance, since $G$ has a polar decomposition $G=K \exp (i \mathfrak{k}))$. The $A v\left(f_{i}\right)$ are also of degree $<r$, and so we may assume, by an induction on $r$, that we have already shown that they are generated by the $s_{i}$ in $R_{+}^{G}$. Thus $s$ is also.

Thus we simply define $X / G$ to be $\operatorname{Proj} \bigoplus_{r} H^{0}(X, \mathcal{O}(r))^{G}$. If $X$ is a variety (rather than a scheme) then so is $X / G$, as its graded ring sits inside that of $X$ and so has no zero divisors. Unfortunately this is not all there is to GIT, however. We have to work out what $X / G$ is, which orbits points of $X / G$ represent, and so on, which we tackle in the next section. Another important question, that we barely touch on, is how the quotient $X / G$ changes with the linearisation. For some linearisations the quotient is empty, but if under a change of linearisation the moduli space remains the same dimension then it undergoes only a small birational transformation, a type of flip [DH, Th].

The affine case. The affine case is even easier; if $G$ acts on Spec $R$ we can form $\operatorname{Spec}\left(R^{G}\right)$ as a putative quotient. For instance in our example (3.2) the ring of invariants

$$
R^{G}=\mathbb{C}[x, y]^{\mathbb{C}^{*}}=\mathbb{C}[x y]
$$

is generated by $x y$, so that the quotient is $\operatorname{Spec} \mathbb{C}[x y] \cong \mathbb{C}$, as anticipated. The function $x y$ does not distinguish between any of the "bad" orbits (the punctured $x$ - and $y$ - axes, and the origin), lumping them all in an equivalence class of orbits which get identified in the quotient.

In other cases this does not work so well; for instance under the scalar action of $\mathbb{C}^{*}$ on $\mathbb{C}^{n+1}$ the only invariant polynomials in $\mathbb{C}\left[x_{0}, \ldots, x_{n}\right]$ are the constants and this recipe for the quotient gives a single point. In the language of the next section, this is because there are no stable points in this example, and all semistable orbits' closures intersect (or equivalently, there is a unique polystable point, the origin). More generally in any affine case all points are always at least semistable (as the constants are always $G$-invariant functions) and so no orbits gets thrown away in making the quotient (though many may get identified with each other - those whose closures intersect which therefore cannot be separated by invariant functions). But for the 
scalar action of $\mathbb{C}^{*}$ on $\mathbb{C}^{n+1}$ we clearly need to remove at least the origin to get a sensible quotient.

So we should change the linearisation, from the trivial linearisation to a nontrivial one, to get a bigger quotient.

Example: $\mathbb{P}^{n}$ from GIT. That is, we consider the trivial line bundle on $\mathbb{C}^{n+1}$ but with a nontrivial linearisation, by composing the $\mathbb{C}^{*}$-action on $\mathbb{C}^{n+1}$ by a character $\lambda \mapsto \lambda^{-p}$ of $\mathbb{C}^{*}$ acting on the fibres of the trivial line bundle over $\mathbb{C}^{n+1}$. The invariant sections of this no longer form a ring; we have to take the direct sum of spaces of sections of all powers of this linearisation, just as in the projective case, and take Proj of the invariants of the resulting graded ring.

If $p<0$ then there are no invariant sections and the quotient is empty. We have seen that for $p=0$ the quotient is a single point. For $p>0$ the invariant sections of the $k$ th power of the linearisation are the homogeneous polynomials on $\mathbb{C}^{n}$ of degree $k p$. So for $p=1$ we get the quotient

$$
\mathbb{C}^{n+1} / \mathbb{C}^{*}=\operatorname{Proj} \bigoplus_{k \geq 0}\left(\mathbb{C}\left[x_{0}, \ldots, x_{n}\right]_{k}\right)=\operatorname{Proj} \mathbb{C}\left[x_{0}, \ldots, x_{n}\right]=\mathbb{P}^{n}
$$

For $p \geq 1$ we get the same geometric quotient but with the line bundle $\mathcal{O}(p)$ on it instead of $\mathcal{O}(1)$.

Another way to derive this is to embed $\mathbb{C}^{n+1}$ in $\mathbb{P}^{n+1}$ as $x_{n+1}=1$, act by $\mathbb{C}^{*}$ on the latter by $\operatorname{diag}\left(\lambda, \ldots, \lambda, \lambda^{-(n+1)}\right) \in S L(n+2, \mathbb{C})$, and do projective GIT. This gives, on restriction to $\mathbb{C}^{n+1} \subset \mathbb{P}^{n+1}$, the $p=n+1$ linearisation above. The invariant sections of $\mathcal{O}((n+2) k)$ are of the form $x_{n+1}^{k} \cdot f$, where $f$ is a homogeneous polynomial of degree $(n+1) k$ in $x_{1}, \ldots, x_{n}$. Therefore the quotient is

$$
\operatorname{Proj} \bigoplus_{k \geq 0}\left(\mathbb{C}\left[x_{1}, \ldots, x_{n}\right]_{(n+1) k}\right)=\left(\mathbb{P}^{n}, \mathcal{O}(n+1)\right) .
$$

In the language of the next section this is because the complement of $\mathbb{C}^{n+1} \subset$ $\mathbb{P}^{n+1}$, and the origin $\{0\} \in \mathbb{C}^{n+1} \subset \mathbb{P}^{n+1}$, are unstable (either by noting that all of the nonconstant invariant polynomials above vanish on them, or by an easy exercise in using the Hilbert-Mumford criterion below - these loci are fixed points, but with a nontrivial action on the line above them). So these are removed and the projective quotient reduces to the affine case.

What are the points of a GIT quotient? By its very definition (and Lemma 3.3), for $r \gg 0, X / G$ is just the image of $X$ under the linear system $H^{0}\left(\mathcal{O}_{X}(r)\right)^{G}$. That is, consider the Kodaira "embedding" of $X / G(2.4)$,

$$
\begin{array}{rrrr}
X & -\rightarrow & \mathbb{P}\left(\left(H^{0}(X, \mathcal{O}(r))^{G}\right)^{*}\right), & \\
x & \mapsto \quad e v_{x} & \left(e v_{x}(s):=s(x)\right),
\end{array}
$$

that in coordinates takes $x$ to $\left(s_{0}(x): \ldots: s_{k}(x)\right) \in \mathbb{P}^{k}$ (where the $s_{i}$ form a basis for $H^{0}(X, \mathcal{O}(r))^{G}$ ). 
This is only a rational map, since it is only defined on points for which $e v_{x} \not \equiv 0$ (equivalently the $s_{i}(x)$ are not all zero). That is, it is defined on the semistable points of $X$ :

Definition 3.6. $x \in X$ is semistable if and only if there exists $s \in$ $H^{0}(X, \mathcal{O}(r))^{G}$ with $r>0$ such that $s(x) \neq 0$.

Points which are not semistable we call (controversially) unstable.

So semistable points are those that the $G$-invariant functions "see". The map (3.5) is well defined on the (Zariski open, though possibly empty) locus $X^{s s} \subseteq X$ of semistable points, and it is clearly constant on $G$-orbits, i.e. it factors through the set-theoretic quotient $X^{s s} / G$. But it may contract more than just $G$-orbits, so we need another definition.

Definition 3.7. A semistable point $x$ is stable if and only if $\bigoplus_{r} H^{0}(X, \mathcal{O}(r))^{G}$ separates orbits near $x$ and the stabiliser of $x$ is finite.

By "separates orbits near $x$ " we mean the following. Since $x$ is semistable there exists an $s \in H^{0}\left(\mathcal{O}_{X}(r)\right)^{G}$ such that $s(x) \neq 0$. So now we work on the open set $U \subset X$ on which $s \neq 0$ and use $s$ to trivialise $\mathcal{O}_{U}(r)$ (i.e., divide all sections of $\mathcal{O}_{U}(r)$ by $s$ to consider them as functions). Then we ask that in $U$ any orbit can be distinguished from $G . x$ by $H^{0}(X, \mathcal{O}(r))^{G}$. That is, there is an element of $H^{0}(X, \mathcal{O}(r))^{G}$ which takes different values on the two orbits, and this should also be true infinitesimally: given a vector $v \in T_{x} X \backslash T_{x}(G . x)$, there is an element of $H^{0}(X, \mathcal{O}(r))^{G}$ whose derivative down $v$ is nonzero.

So we have a (surjective) map $X^{s s} \rightarrow X / G$ under which the line bundle on $X / G$ (that arises from its Proj construction) pulls back to $\mathcal{O}_{X^{s s}}(1)$. This map has good geometric properties over the locus of stable points $X^{s} \subseteq$ $X^{s s} \subseteq X$ (it only contracts single orbits, for instance; more properly it is a geometric quotient in the sense of [GIT, Definition 0.6]). The definitions of stable and semistable are the algebraic ones designed to make this true, but now we can relate them more to geometry.

Topological characterisation of (semi)stability. If we work upstairs in the vector space $\mathbb{C}^{n+1} \supset \widetilde{X}$ (or equivalently in the total space of $\mathcal{O}_{X}(-1)$ ) instead of in the projective space $\mathbb{P}^{n} \supset X$, we can get a beautiful topological characterisation of (semi)stability. Given our topological discussion about nonclosed orbits at the start of these notes, it is what one might guess, and the best one could possibly hope for. So for $x \in X$, pick $\tilde{x} \in \mathcal{O}_{X}(-1)$ covering it.

THEOREM 3.8.

$x$ is semistable $\Longleftrightarrow 0 \notin \overline{G . \tilde{x}}$.

$x$ is stable $\Longleftrightarrow G . \tilde{x}$ is closed in $\mathbb{C}^{n+1}$ and $\tilde{x}$ has finite stabiliser.

When G. $\tilde{x}$ is closed, but not necessarily of full dimension, we call $x$ polystable. (This is called "Kempf-stable" in [Dl], "weakly stable" in 
[Ti4] and plain "stable" in [Do5]. Originally [GIT] the terms for stable and polystable were "properly stable" and "stable" respectively.)

In one direction the theorem is clear. $G$-invariant homogeneous functions of degree $r>0$ on $\widetilde{X}$ are constant on orbits and so also their closures. So if the closure of the orbit of $\tilde{x}$ contains the origin then every such function is zero on $\widetilde{x}$ and $x$ cannot be semistable. Similarly if the invariant functions separate orbits around the orbit of $\tilde{x}$ then it is the zero locus of a collection of invariant functions and so closed.

One can make the criterion (3.8) much simpler to calculate with, by considering one parameter subgroups instead of all of $G$.

The Hilbert-Mumford criterion. The key result is that $x$ is (semi)stable for $G$ if and only if it is (semi)stable for all one parameter subgroups $(1-P S s) \mathbb{C}^{*}<G$. We will outline a proof of this remarkable result once we have done some symplectic geometry.

So we may apply Theorem 3.8 to each of these 1-PS orbits, and determining the closedness of these one dimensional orbits is much easier by using their asymptotics. Setting $x_{0}=\lim _{\lambda \rightarrow 0} \lambda . x$, this is a fixed point of the $\mathbb{C}^{*}$-action, so $\mathbb{C}^{*}$ acts on the line $\mathcal{O}_{x_{0}}(-1)$ in $\mathbb{C}^{n+1}$ that $x_{0} \in \mathbb{P}^{n}$ represents. Letting $\rho(x) \in \mathbb{Z}$ denote the weight of this action (i.e. $\mathbb{C}^{*} \ni \lambda$ acts on $\mathcal{O}_{x_{0}}(-1)$ as $\left.\lambda^{\rho(x)}\right)$ we find the following, the Hilbert-Mumford criterion.

\section{THEOREM 3.9.}

- If $\rho(x)<0$ for all 1 -PS then $x$ is stable,

- If $\rho(x) \leq 0$ for all 1 -PS then $x$ is semistable,

- If $\rho(x)>0$ for a 1 -PS then $x$ is unstable.

The proof is the picture below; the 1-PS orbit is closed if and only if it is asymptotic to a negative weight $\mathbb{C}^{*}$-action on the limiting line at both $\lambda \rightarrow 0$ and $\lambda \rightarrow \infty$. But we can restrict to the former since the latter arises from the inverse 1-PS.
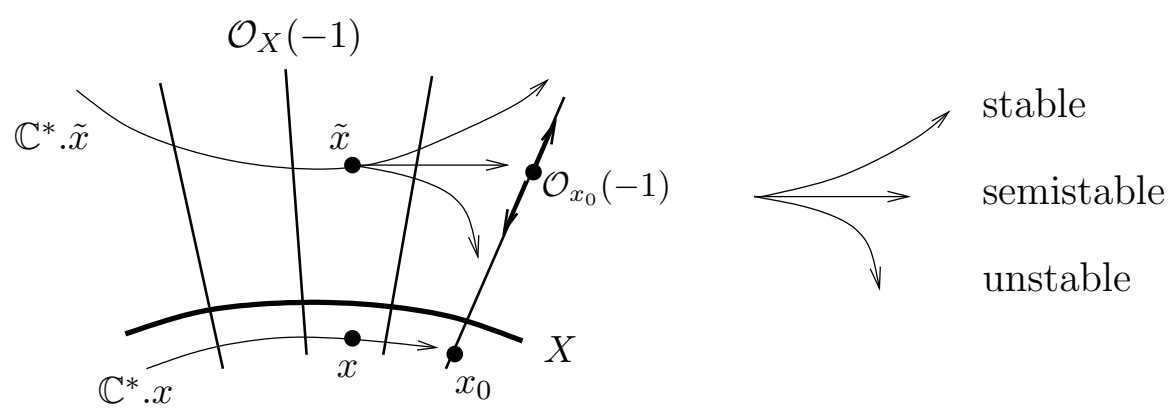

So we "just" have to compute the weight $\rho(x)$ for all $\mathbb{C}^{*}<S L(n+1, \mathbb{C})$; $x$ is stable for $G$ if and only if $\rho(x)$ is always $<0$.

To sum up; the 1-PS orbits are not (in general) closed in the projective space, but they may be upstairs in the vector space. To decide if $x$ is stable 
we first take the limit $x_{0}$ as we move through isomorphic objects $\lambda . x$; this limit is not (in general) isomorphic to $x$ under the $\mathbb{C}^{*}$-action; it is only in the closure of the $\mathbb{C}^{*}$-orbit. This point $x_{0}$ represents a line $\mathcal{O}_{x_{0}}(-1)$ in the vector space, on which $\mathbb{C}^{*}$ acts. If this weight is negative then $x$ (not $x_{0}$ !) is stable for this 1-PS; if this is true for all 1-PSs then $x$ is stable for $G$.

Fundamental example: points in $\mathbb{P}^{1}$. The standard example, from for instance [GIT], is to consider configurations of $n$ (unordered) points in $\mathbb{P}^{1}$ up to the symmetries of $\mathbb{P}^{1}$. (This is of course a 0-dimensional algebraic variety, and so the easiest example of the stability of varieties that we shall study later.) In fact we allow multiplicities, i.e. we take length- $n$ 0-dimensional subschemes of $\mathbb{P}^{1}-S^{n} \mathbb{P}^{1}-$ modulo $S L(2, \mathbb{C})$.

To linearise the action we note that specifying any such $n$ points is the same as specifying a degree $n$ homogeneous polynomial on $\mathbb{P}^{1}$, unique up to scale, by taking the roots of the polynomial. That is, $S^{n} \mathbb{P}^{1}$ is the projectivisation of $H^{0}\left(\mathcal{O}_{\mathbb{P}^{1}}(n)\right)$, so giving us a natural linearisation of the problem; we use the induced $S L(2, \mathbb{C})$-action on $H^{0}\left(\mathcal{O}_{\mathbb{P}^{1}}(n)\right) \cong S^{n}\left(\mathbb{C}^{2}\right)^{*}$.

We find that the configuration is stable unless it has a very singular point.

THEOREM 3.10. A length-n subscheme of $\mathbb{P}^{1}$ is

- semistable if and only if each multiplicity $\leq n / 2$,

- stable if and only if each multiplicity $<n / 2$.

Proof. 1. Diagonalise a given $\mathbb{C}^{*}<S L(2, \mathbb{C})$ :

$$
\left(\begin{array}{cc}
\lambda^{k} & 0 \\
0 & \lambda^{-k}
\end{array}\right) \text { in }[x: y] \text { coordinates on } \mathbb{P}^{1} \cdot(k \geq 0 .)
$$

In these coordinates write our degree $n$ homogeneous polynomial (whose roots give the $n$ points) as $f=\sum_{i=0}^{n} a_{i} x^{i} y^{n-i}$. As $\lambda \rightarrow 0$ the first half (precisely the first $\lceil n / 2-1\rceil$ ) of these monomials tend to infinity (as there are more $y$ s than $x$ s in the monomial).

Thus $\lambda$. $f$ tends to $\infty$ and the orbit is closed about $\lambda \rightarrow 0$ unless $a_{i}=0$ for $i \leq n / 2$. That is, it is closed so long as $f$ does not vanish to order $\geq n / 2$ at $x=0$.

Repeating over all 1-PS changes the coordinates $[x: y]$, so $f$ is stable if and only if it does not vanish to order $\geq n / 2$ at any point.

Alternatively, we can use the Hilbert-Mumford criterion in terms of the weight on the limiting line.

Proof. 2. Up to rescaling, under the action (3.11), $\lambda . f \rightarrow f_{0}=$ $a_{j} x^{j} y^{n-j}$, where $j$ is smallest such that $a_{j} \neq 0$.

The weight of $(3.11)$ on $\mathbb{C} . f_{0}$ is $k(j-(n-j))=k(2 j-n)$. So $f$ is stable if and only if $k(2 j-n)<0 \Longleftrightarrow j<n / 2 \Longleftrightarrow \operatorname{ord}_{x=0}(f)<n / 2$ for all 1 -PS (and so all points $x=0$ ) as before. Semistability is similar. 


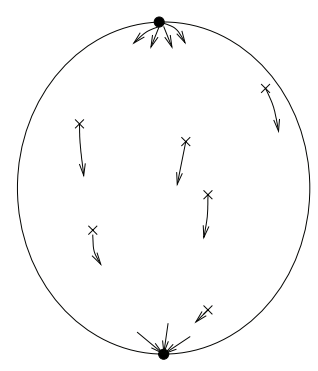

Geometrically what is happening is that the 1-PS moves almost all points to the "attractive" fixed point at $x=0$ (weight $-k$ ), and this is the generic, stable, situation. Only those points stuck at "repulsive" fixed point $y=0$ contribute positively $(+k)$ to the weight. So the total weight is negative unless more than half of the points are at the repulsive fixed point.

More generally if we consider hypersurfaces $D \subset \mathbb{P}^{n}$ modulo the action of $S L(n+1, \mathbb{C})$, then the existence of the discriminant of the defining equation (which is automatically an invariant polynomial) means that

$$
D \text { smooth } \Rightarrow D \text { semistable, }
$$

since its discriminant does not vanish.

Another topological characterisation of stability. Instead of working in $\widetilde{X}$, we can give a topological characterisation of stability (not semistability) downstairs in $X$ which, in the language of the first section, says that we have removed enough orbits to get a Hausdorff quotient.

Namely, polystability of $x$ is equivalent to the orbit of $x$ being closed in the locus of semistable points.

For stability, one direction is clear; by the original definition of stability of $x$, the closed locus where the invariant sections $\bigoplus_{k} H^{0}\left(L^{k}\right)^{G}$ take the value that they take on $G . x$ is precisely $G . x$.

For the converse we note that given any 1-PS orbit of a stable point $x$, its limit point $x_{0}$ is unstable, by the Hilbert-Mumford criterion applied to the inverse 1-PS (under which $x_{0}$ is fixed and $\mathcal{O}_{x_{0}}(-1)$ is acted on with positive weight). Thus once $x_{0}$ is removed, i.e. in the locus of semistable points, the 1-PS orbit of $x$ is closed (at the $\lambda \rightarrow 0$ end; then one can consider the inverse 1-PS). Then one has to show that this enough to show that the whole of $G . x$ is closed in $X^{s s}$.

The polystable case follows by showing that for any semistable point $x$,

$$
\bigcap\{\overline{G . y}: \overline{G . y} \cap \overline{G . x} \neq \emptyset\}
$$

is a single orbit, which is therefore closed. This is the unique polystable representative of the semistable equivalence class of orbits which are identified together in the GIT quotient. This can be proved using the Kempf-Ness theorem in a later Section. 
Another form of the Hilbert-Mumford criterion. The HilbertMumford criterion can be recast in terms of $\tilde{x}$ only (with no mention of $x_{0}$ ) in a form that can be useful in calculations.

In our usual set-up of $G$ acting on $X \subseteq \mathbb{P}^{n}$ linearised over $\mathcal{O}_{X}(-1)$, any 1-PS therefore gives a 1 -PS of $S L(n+1, \mathbb{C})$ (in fact $G L(n+1, \mathbb{C})$, but it is an easy exercise to see that if it does not lie in $S L(n+1, \mathbb{C})$ then all points are unstable).

Diagonalising we can write the action as $\lambda \mapsto \operatorname{diag}\left(\lambda^{\rho_{0}}, \ldots, \lambda^{\rho_{n}}\right)$, in which basis $\tilde{x}=\left(x_{0}, \ldots, x_{n}\right)$. Then it is clear that the orbit $\{\lambda . \tilde{x}\}$ tends to $\infty$ as $\lambda \rightarrow 0$ if and only if there is an $i$ such that

$$
x_{i} \neq 0 \text { and } \rho_{i}<0 .
$$

This is true for all 1-PSs if and only if $\tilde{x}$ is stable. The semi- and polystable cases are left as an exercise for the reader.

So the vector space $\mathbb{C}^{n+1}=W^{+} \oplus W^{0} \oplus W^{-}$can be split into a sum of positive and negative weight spaces (with the sum of all weights, with multiplicities, zero) for the $\mathbb{C}^{*}<S L(n+1, \mathbb{C})$. The condition that $\tilde{x}$ be stable with respect to this 1-PS and its inverse is then that its components in both of $W^{+}$and $W^{-}$be nonzero; i.e., that it have components of both positive and negative weight. Hence generic points are stable if the group acts effectively through $S L(n+1, \mathbb{C})$.

\section{Symplectic reduction}

In this section we use the compact subgroup $K<G$ to enlarge (3.1) to

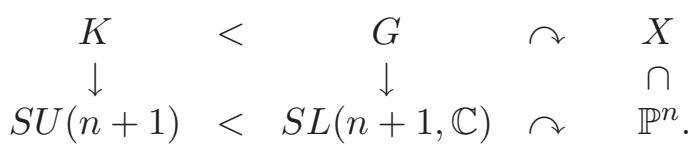

So $K$ acts on $\mathbb{P}^{N}$, preserving the complex structure $J$ (as $S L(n+1, \mathbb{C})$ does) but also the Fubini-Study metric $g$. Thus $K$ preserves the symplectic form $\omega=g(\cdot, J \cdot)$ and acts through symplectomorphisms in $\operatorname{Aut}(X, \omega)$.

Hamiltonian automorphisms. The Lie algebra of the symplectomorphism group consists of vector fields $Y$ on $X$ which preserve the symplectic form, i.e.

$$
\left.\mathcal{L}_{Y} \omega=d(Y\lrcorner \omega\right)=0 .
$$

Since contraction with $\omega$ is an isomorphism $T X \stackrel{\simeq}{\longrightarrow} T^{*} X$, the Lie algebra is isomorphic to the space of closed 1 -forms $Z^{1}(X)$.

The subspace of exact 1-forms $d C^{\infty}(X, \mathbb{R})$ (which is all of them for simply connected spaces like $\mathbb{P}^{N}$ ) generate hamiltonian automorphisms - those which can be connected to the identity through a path of automorphisms whose flux homomorphism is zero (i.e., integration of $\omega$ over the cylinder traced out in this path by any loop in $X$ is zero). 
Since $d$ has kernel the constants, the Lie algebra of the hamiltonian automorphisms is $C^{\infty}(X, \mathbb{R}) / \mathbb{R}$; the function $h$ generating the vector field

$$
\left.X_{h} \text { such that } X_{h}\right\lrcorner \omega=d h .
$$

That is, using the metric, $X_{h}$ is the symplectic gradient $J \nabla h$ of the hamiltonian $h$.

The Lie bracket of hamiltonian vector fields works out to be the Poisson bracket $\{f, g\}$ on functions, given by pairing $d f$ and $d g$ using the (inverse of) the symplectic form and dividing out by constants. (Equivalently, this is the class of $X_{f}(g)=-X_{g}(f)$ in $C^{\infty}(X, \mathbb{R}) / \mathbb{R}$.)

This bracket clearly lifts to $C^{\infty}(X, \mathbb{R})$, and can be checked to satisfy the Jacobi identity there too. The constants are central - they Poisson commute with all of $C^{\infty}(X, \mathbb{R})$ - so we get a central extension of the Lie algebra of hamiltonian automorphisms:

$$
0 \rightarrow \mathbb{R} \rightarrow C^{\infty}(X, \mathbb{R}) \rightarrow C^{\infty}(X, \mathbb{R}) / \mathbb{R} \rightarrow 0 .
$$

One might ask what $C^{\infty}(X, \mathbb{R})$ is the Lie algebra of, or what it acts on infinitesimally. Since $\mathbb{R}$ is the Lie algebra of isometries of the line $\mathbb{C}$, one might consider isometries of a line bundle $L \rightarrow X$ covering hamiltonian automorphisms on $X$.

This indeed can be made to work if $\omega$ is integral, i.e. its cohomology class lies in $H^{2}(X, \mathbb{Z}) /$ torsion $\leq H^{2}(X, \mathbb{R}$ ) (as in our projective case, for instance). Then $2 \pi i \omega$ is the curvature of a hermitian line bundle $L$ with unitary connection, and we let $\widetilde{\operatorname{Ham}}(X, \omega)$ be the isometries of $L$ preserving its connection; these then cover hamiltonian automorphisms on $X$. Infinitesimally $h \in C^{\infty}(X, \mathbb{R})$ acts through vector fields on $L$ given by

$$
\tilde{X}_{h}+i h .
$$

Here $\tilde{X}_{h}$ is the horizontal lift of the hamiltonian vector field $X_{h}$, and $i h$ is the multiplication operator taking element of the line $L$ to a perpendicular element in its tangent space (using the natural isomorphism between a line $L$ and its tangent space). This action defines a homomorphism of Lie algebras (the Poisson bracket on $C^{\infty}(X, \mathbb{R})$ maps to the Lie bracket on vector fields on the total space of $L$ ), and the constants $\mathbb{R}$ act as the Lie algebra of global constant rotations $\left\{e^{i \theta}\right\}=U(1)$ of the fibres of $L$, yielding the exact sequence $(4.2)$.

(This is often called prequantisation, giving a representation of the hamiltonian diffeomorphisms on the projectivisation of the Hilbert space of $L^{2}$ sections of $L$. This is considered too big a Hilbert space to be the set of quantum mechanical wave functions, and geometric quantisation attempts to replace it with holomorphic sections; about which more later.)

Moment maps and linearisations. Since $K$ acts through symplectomorphisms of $\mathbb{P}^{n}$, which is simply connected (so that $Z^{1}(X)=d C^{\infty}(X)$ ), 
we get a Lie algebra homomorphism

$$
\mathfrak{k}=\operatorname{Lie}(K) \rightarrow C^{\infty}\left(\mathbb{P}^{n}, \mathbb{R}\right) / \mathbb{R} \rightarrow C^{\infty}(X, \mathbb{R}) / \mathbb{R}, \quad v \mapsto\left[m_{v}\right],
$$

where any $v \in \mathfrak{k}$ generates a hamiltonian vector field $X_{v}$ on $X$ such that $\left.X_{v}\right\lrcorner \omega=d m_{v}$, for some function $m_{v}$ unique up to a constant.

We would like to choose these constants consistently, i.e., choose a lift
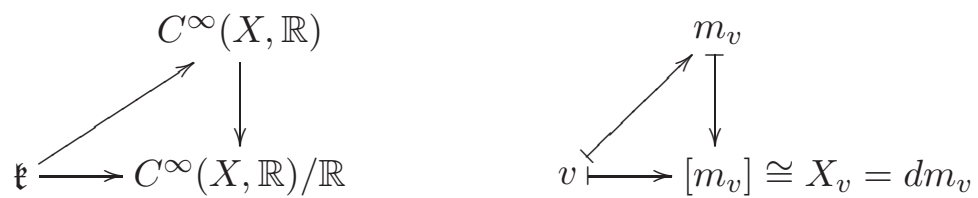

which is a homomorphism of Lie algebras. One such always exists, since (4.2) is split by the Poisson subalgebra $C^{\infty}(X, \mathbb{R})_{0} \cong C^{\infty}(X, \mathbb{R}) / \mathbb{R}$ of functions of integral zero; i.e., we can choose each $m_{v}$ to have integral zero. But we want to consider arbitrary lifts since from (4.3) we know that each is equivalent to a lift of $\mathfrak{k}$ to isometries of $L$ preserving the connection and covering its hamiltonian action downstairs. This is the infinitesimal version of a linearisation, assigning to $v \in \mathfrak{k}$ the vector field

$$
\tilde{X}_{v}+i m_{v}
$$

on the total space of $L$. It may or may not integrate up to an action of $K$ on $(X, L)$ covering that on $X$.

Said differently, we want to put together all of the hamiltonians $m_{v}$ to give a moment map

$$
m: X \rightarrow \mathfrak{k}^{*},
$$

such that $\langle m(x), v\rangle=m_{v}(x)$ for all $v \in \mathfrak{k}$. $m$ is just a collection of $\operatorname{dim} K$ hamiltonians $m_{v}$, written invariantly. Then our lifting condition (4.4) becomes the condition that the undetermined constants in $m_{v}$ be chosen such that (4.6) is $K$-equivariant (using the coadjoint action on the right hand side). Thus a moment map is unique up to the addition of a central element of $\mathfrak{k}^{*}$.

Yet another way of saying the same thing is that the derivative of the $K$ action maps $\mathfrak{k}$ to $T X$, so by contraction with the symplectic form $\lrcorner \omega: T X \cong$ $T^{*} X$ is a section of $\mathfrak{k}^{*} \otimes T^{*} X$. It is closed and $K$-invariant, so we ask for it to be invariantly exact, i.e. $d$ of a $K$-invariant section $\mu$ of $C^{\infty}\left(X, \mathfrak{k}^{*}\right)$.

(The name comes from the case of a cotangent bundle $X=T^{*} M$ with its canonical symplectic form and action induced from an action of $K$ on $M$. Then the moment map really gives the momentum of the image $X_{v} \in T M$ of $v \in \mathfrak{k}: m_{v}(p, q)=\left\langle p, X_{v}\right\rangle$ at a point $q \in M$ and $p \in T_{q}^{*} M$. Hence for translations we get the usual linear momentum, and for rotations angular momentum.)

In the projective case that we have been considering, a natural $m$ exists because we picked a linearisation. $S U(n+1) \curvearrowright\left(\mathbb{P}^{n}, \mathcal{O}(1)\right)$ has a canonical 
moment map given by

$$
\tilde{x} \mapsto \frac{i(\langle\cdot, \tilde{x}\rangle \otimes \tilde{x})_{0}}{\|\tilde{x}\|^{2}} \in \mathfrak{s} u(n+1)^{*} \cong \mathfrak{s} u(n+1),
$$

where ( ) denotes the trace-free part of an endomorphism. Restricting to $X$ and projecting to $\mathfrak{k}^{*}$ by the adjoint of the map $\mathfrak{k} \rightarrow \mathfrak{s} u(n+1)$ gives a moment map for the $K$-action on $X$.

The Kempf-Ness theorem. The key to the link between symplectic geometry and GIT is the following calculation. Suppose $\left(X, L=\mathcal{O}_{X}(1)\right)$ is a polarised variety with a hermitian metric on $L$ inducing a connection with curvature $2 \pi i \omega$. Lift $x$ to any $\tilde{x} \in \mathcal{O}_{x}(-1)=L_{x}^{-1}$ and consider the norm functional $\|\tilde{x}\|$. (If $X$ is embedded in $\mathbb{P}\left(H^{0}(L)^{*}\right)$ then one way to get a metric on $\mathcal{O}(-1)$ is to induce it from one on $H^{0}(L)^{*}$ upstairs; then $\|\tilde{x}\|$ is just the usual norm in the vector space that $\widetilde{X}$ lives in.) As we move down a 1-PS orbit $\left\{\lambda . \tilde{x}: \lambda \in \mathbb{C}^{*}\right\}$ in the direction of $v \in \mathfrak{k}$ we see how $\log \|\tilde{x}\|$ varies; for $\lambda \in U(1)<\mathbb{C}^{*}$ (which preserves the metric) not at all, but for $\lambda$ in the complexified, radial direction $\lambda \in(0, \infty)<\mathbb{C}^{*}$ we get

$$
m_{v}=\left.\frac{d}{d \lambda}\right|_{\lambda=1} \log \|\left.\lambda \tilde{x}\right|_{\lambda \in(0, \infty)} .
$$

That is, $X_{v}(\log \|\lambda \tilde{x}\|)=0$, but

$$
\left(J X_{v}\right)(\log \|\lambda \tilde{x}\|)=X_{i v}(\log \|\lambda \tilde{x}\|)=m_{v} .
$$

(This is just an unravelling of (4.5). For instance if $x$ is a fixed point, then $\mathbb{C}^{*}$ acts on the line $\langle\tilde{x}\rangle$ with a weight $\rho$, and

$$
m_{v}=\rho,
$$

which is therefore an integer.)

Moreover, $\log \|\lambda \tilde{x}\|$ is convex on $\mathbb{C}^{*} / U(1) \cong(0, \infty)$, as its second derivative is positive:

$$
X_{i v} m_{v}=d m_{v}\left(J X_{v}\right)=\omega\left(X_{v}, J X_{v}\right)=\left\|X_{v}\right\|^{2} .
$$

It follows that the orbit tends to infinity at both ends, i.e., is closed, if and only if it contains a critical point (i.e. absolute minimum) of $\log \|\lambda \tilde{x}\|$.

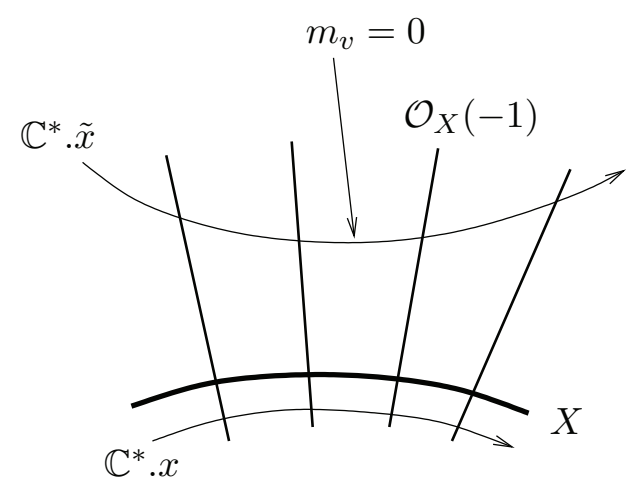


So a 1-PS orbit is polystable if and only if it contains a zero of the corresponding hamiltonian. That zero is then unique, up to the action of $U(1)$. This is the Kempf-Ness theorem for $\mathbb{C}^{*}$-actions.

Next we would like to consider a full $G$ orbit, and find a zero of all the hamiltonians simultaneously, i.e. a zero of $m$. Pick $v_{i}$ to form a basis for the Lie algebra of a maximal torus in $K$ such that each generates a 1-PS. If an orbit is polystable then each 1-PS orbit is closed, so by the above there is a point with $m_{v_{1}}=0$ in the first 1-PS. Now we move down the second 1-PS orbit of this point to a point with $m_{v_{2}}=0$ and $m_{v_{1}}=0$ since the two 1-PSs commute (i.e., $\left\{m_{v_{1}}, m_{v_{2}}\right\}=0$ ). Inductively we find a point with $m_{v}=0$ for all $v$ in the Lie algebra of the torus, and so for all $v$ conjugate to such (i.e. all $v$ ) by equivariance of the moment map. Thus the orbit contains a point with $m=0$. Moreover, by the convexity of $\log \|\tilde{x}\|$ on $G / K$, the zero is in fact unique up to the action of $K$.

(Alternatively, we could have proved this without using the HilbertMumford criterion by noting that $\log \|g . \tilde{x}\|$ is convex on the whole of $G / K$, instead of each $\mathbb{C}^{*} / U(1)$, so an orbit is closed if and only if this functional has a minimum, at which point $m=0$ by (4.8).)

TheOREM 4.11. [Kempf-Ness] A G-orbit contains a zero of the moment map if and only if it is polystable. It is unique up to the action of $K$.

$A G$-orbit is semistable if and only if its closure contains a zero of the moment map; this zero is in the unique polystable orbit in the closure of the original orbit.

In particular, as sets,

$$
\frac{X}{G} \cong \frac{m^{-1}(0)}{K}=: X / / K .
$$

$X / / K:=m^{-1}(0) / K$ is called the symplectic reduction of $X$, invented by Marsden-Weinstein and Meyer.

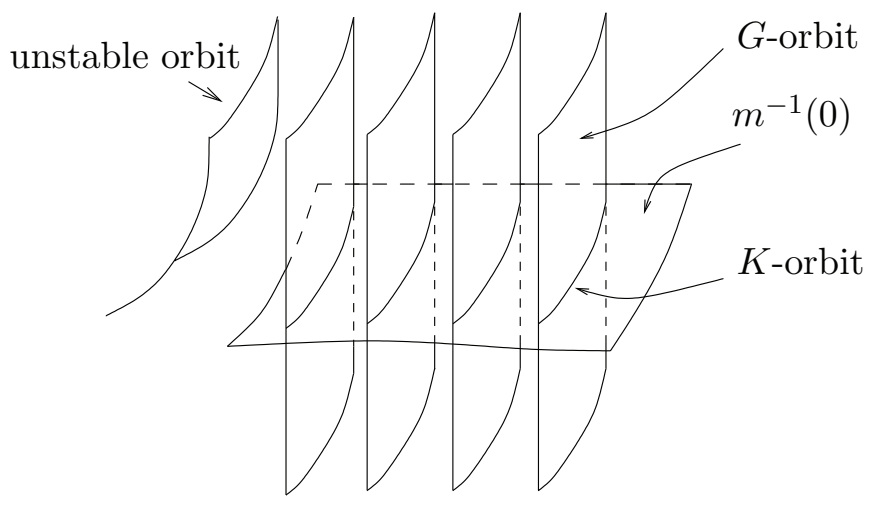

So on the locus of stable points $m^{-1}(0)$ provides a ( $K$-equivariant) slice to the $i \mathfrak{k}<\mathfrak{g}=\mathfrak{k}+i \mathfrak{k}$ part of orbit; since this is topologically trivial ( $G$ retracts onto $K$ ) it makes topological sense that one could take a slice instead 
of a quotient. This leaves only the $K$-action to divide by to get the GIT quotient.

The Kempf-Ness theorem is a nonlinear generalisation of the isomorphism $V / W \cong W^{\perp}$ for vector spaces $W \leq V$. It works due to convexity, giving a unique distinguished $K$-orbit of points of least norm in each polystable $G$-orbit upstairs in $\widetilde{X}$.

When 0 is a regular value of $m$ (which implies that $m^{-1}(0)$ is smooth and the $\mathfrak{k}$-action on it is injective, so the $K$-action has finite stabilisers and the quotient is a smooth orbifold at worst) then the restriction of $\omega$ to $m^{-1}(0)$ is degenerate precisely along the $K$-orbits, and so descends to a symplectic form on the quotient. This is in fact compatible with the complex (algebraic) structure on the GIT quotient, giving a Kähler form representing the first Chern class of the polarisation that $X / G$ inherits from its Proj construction.

Example. $U(1)<\mathbb{C}^{*}$ acts on $\mathbb{C}^{n+1}$ with moment map $m=|\underline{z}|^{2}-a$ for any constant $a \in \mathbb{R}$. For $a>0$ this gives

$$
\frac{\mathbb{C}^{n+1} \backslash\{0\}}{\mathbb{C}^{*}} \cong \frac{S^{2 n+1}=\left\{\underline{z}:|\underline{z}|^{2}=a\right\}}{U(1)} \cong \mathbb{P}^{n}
$$

$S^{2 n+1}=m^{-1}(0)$ is a slice to the $(0, \infty)$-action, leaving the $U(1)$-action to divide by. The resulting Kähler form on $\mathbb{P}^{n}$ varies with the level $a$.

For $a=0$ we get just a single point, while for $a<0$ we get the empty set - as we showed already using GIT for different polarisations (3.4), where $p$ played the role of $a$ (but took integer values so that the lifted action of $\mathfrak{k}$ descended to an action of $K=U(1)$ on the trivial line bundle over $\mathbb{C}^{n+1}$ ).

Example: $n$ points in $\mathbb{P}^{1}$ again. (Kirwan $[\mathbf{K i}]$ ) The moment map

$$
S L(2, \mathbb{C}) \supset S U(2) \curvearrowright \mathbb{P}^{1} \stackrel{m}{\longrightarrow} \mathfrak{s} u(2)^{*}
$$

is just the inclusion of the unit sphere $S^{2} \subset \mathbb{R}^{3}$.

Adding gives, for $n$ points, the moment map $m=\sum_{i=1}^{n} m_{i}$ :

$$
S^{n} \mathbb{P}^{1} \longrightarrow \mathbb{R}^{3}
$$

the sum of the $n$ points in $\mathbb{R}^{3}$, i.e., ( $n$ times) their centre of mass.

So $m^{-1}(0)$ is the set of balanced configurations of points with centre of mass $0 \in \mathbb{R}^{3}$.

Since by Kempf-Ness polystability is equivalent to the existence of an $S L(2, \mathbb{C})$ transformation of $\mathbb{P}^{1}$ that balances the points, Theorem 3.10 yields

THEOREM 4.13. A configuration of points with multiplicities in the unit sphere $S^{2} \subset \mathbb{R}^{3}$ can be moved by an element of $S L(2, \mathbb{C})$ to have centre of mass the origin if and only if either each multiplicity is strictly less than half the total, or there are only 2 points and both have the same multiplicity.

The first case is the stable case, the second the polystable case with a $\mathbb{C}^{*}$-stabiliser. 
Example: Grassmannians from GIT and symplectic reduction. We have seen how to get $\mathbb{P}^{n}$ by GIT and symplectic reduction; we can do something similar for Grassmannians.

Consider $S L(r, \mathbb{C})$ acting on $\operatorname{Hom}\left(\mathbb{C}^{r}, \mathbb{C}^{n}\right), r<n$, linearising the induced action on the projectivisation $\mathbb{P}$ of this vector space (we choose the left action of multiplying on the right by $g^{-1}$ ).

Proposition 4.14. $[A] \in \mathbb{P}$ is stable if $A \in \operatorname{Hom}\left(\mathbb{C}^{r}, \mathbb{C}^{n}\right)$ has full rank $r$, and unstable otherwise.

Proof. If $\operatorname{rank}(A)<r$ then we can pick a splitting $\mathbb{C}^{r}=\langle v\rangle \oplus W$ with $A(v)=0$. Then the 1-PS that acts as $\lambda^{r-1}$ on $v$ and $\lambda^{-1}$ on $W$ fixes $[A] \in \mathbb{P}$ and acts on the line $\mathbb{C}$. $A$ with weight +1 . Therefore $[A]$ is unstable by the Hilbert-Mumford criterion.

Conversely, if $A$ has full rank then, up to the action of $S L(r, \mathbb{C})$ some multiple of it is the inclusion of the first factor of some splitting $\mathbb{C}^{n} \cong$ $\mathbb{C}^{r} \oplus \mathbb{C}^{n-r}$. Diagonalising a given 1-PS, we may assume further that in this basis we have the action

$$
\operatorname{diag}\left(\lambda^{\rho_{1}}, \ldots, \lambda^{\rho_{r}}\right), \quad \rho_{1} \geq \rho_{2} \geq \ldots \geq \rho_{r}, \quad \sum_{i} \rho_{i}=0 .
$$

Ignoring the trivial 1-PS, there is some $p$ such that $\rho_{1}=\rho_{p}>\rho_{p+1}$. Then the limit $\left[A_{0}\right]$ of $[A]$ under this 1-PS is the inclusion of $\mathbb{C}^{p}$ as the first $p$ basis vectors of $\mathbb{C}^{n}$, with the 1 -PS acting with weight $-\rho_{1}<0$ on $\mathbb{C}$. $A_{0}$. Therefore $A$ is stable.

So the points of the GIT quotient are the injections of $\mathbb{C}^{r}$ into $\mathbb{C}^{n}$ modulo the automorphisms of $\mathbb{C}^{r}$; i.e., they are the images of the injections - the Grassmannian $G r(r, n)$ of $r$ dimensional subspaces of $\mathbb{C}^{n}$.

For symplectic reduction, it is easier to consider the affine case of $U(r)<$ $G L(r, \mathbb{C})$ acting on $\operatorname{Hom}\left(\mathbb{C}^{r}, \mathbb{C}^{n}\right)$, with all vector spaces endowed with their standard metrics. (Above, by working with $\mathbb{P}$, we had already divided out by the centre of $G L(r, \mathbb{C})$ but didn't describe it this way because, as we have seen, it is easier to deal with the linearisation issues in the symplectic picture, where it just amounts to changing the moment map by a central scalar.) The moment map is

$$
A \mapsto i\left(A^{*} A-\mathrm{id}\right)
$$

with zeros the orthogonal linear maps that embed $\mathbb{C}^{r}$ isometrically. Thus Kempf-Ness recovers the obvious fact that a linear map is congruent by $G L(r, \mathbb{C})$ to an isometric embedding if and only if it is injective. Dividing these isometric embeddings by $U(r)$ gives $G r(r, n)$ again.

More affine examples. Our simple example (3.2) has moment map

$$
\left(|x|^{2}-|y|^{2}\right) / 2
$$

whose zero set intersects each good orbit $x y=\alpha \neq 0$ in a unique $U(1)$ orbit $\sqrt{\alpha}\left(e^{i \theta}, e^{-i \theta}\right)$. It intersects the origin (another $U(1)$ orbit, corresponding 
to $\alpha=0$ ) and misses the other two orbits (the punctured $x$ - and $y$-axes). Therefore the symplectic quotient is a copy of $\mathbb{C}$ parameterised by $\alpha$, representing the closed, polystable orbits, as anticipated.

If we chose the moment map $\left(|x|^{2}-|y|^{2}+a\right) / 2, a>0$, then we miss the $x$-axis and the origin, and gain a unique $U(1)$ orbit on the $y$-axis. So the symplectic quotient is isomorphic, but with a different interpretation. This corresponds in GIT to a different linearisation, in which the $x$-axis and the origin are unstable and the punctured $y$-axis is stable. (So this nonclosed orbit becomes closed upstairs in the new linearisation, and is closed in the locus of semistable points.)

Another standard example is to consider $n \times n$ complex matrices acted on by the adjoint action of $S L(n, \mathbb{C})$. The invariant polynomials are the symmetric functions in the eigenvalues of the matrix (by the denseness of the set of diagonalisable matrices) - i.e. functions in the coefficients of the characteristic polynomial. This reflects the fact that the matrices with nondiagonal Jordan canonical form have the corresponding diagonal matrices in the closure of their orbits - all matrices are semistable for this linearisation (the constant 1 does not vanish on any orbit!), with the diagonalisable matrices being polystable (their stabiliser is at least $\left(\mathbb{C}^{*}\right)^{n}$, after all).

The moment map (for the standard symplectic structure inherited from $\left.\mathbb{C}^{n^{2}}\right)$ for the induced action of $S U(n)$ is $A \mapsto \frac{1}{2}\left[A, A^{*}\right]$ with zeros the normal matrices. Since normal matrices are those that can be orthogonally diagonalised, the symplectic quotient \{normal matrices\}/SU( $n)$ is the set of diagonal matrices up to the action of the symmetric group, and so equal to the GIT quotient. (So in this case Kempf-Ness is the obvious fact that a matrix can be diagonalised if and only if it is similar to a matrix that can be orthogonally diagonalised.)

Back to the Hilbert-Mumford criterion. For simplicity of exposition we used the Hilbert-Mumford criterion to prove the Kempf-Ness theorem, to reduce everything to single hamiltonians. But as we noted there, we could have avoided this and proved it directly by noting that $\log \|g . \tilde{x}\|$ is convex on the whole of $G / K$, so an orbit is closed if and only if this log-norm functional is proper, in which case it has a minimum, at which point $m=0$ by (4.8).

We can then use this to go back and give a sketch proof (more of a discussion, really) of the Hilbert-Mumford criterion. That is we want to show that properness is equivalent to properness on 1-PSs. As usual one direction is trivial; for the other one can try to work on $G / K$ as in, for instance, $[\mathbf{D K}]$. The idea is that while 1 -PSs cover very little of $G$, since $K$ preserves the norm functional it descends to $G / K$, in which 1-PSs are dense (see the torus case below where the 1 -PSs correspond to directions in $\mathfrak{g} / \mathfrak{k} \cong \mathfrak{k}$ of rational slope). Although it is not a priori clear that properness down each such rational direction is enough to give properness on all of $G / K$, it is clear by openness that if a $G$-orbit is strictly unstable then there will be 
a rational direction (1-PS) that detects it. So we see that (semi)stability of each 1-PS implies semistability for $G$.

So this leaves the hard part - that strict stability for each 1-PS implies strict stability for $G$. That is, we want to show that if a $G$-orbit is strictly semistable, then there is a 1-PS with zero weight; i.e. the non-properness is detected by a rational direction.

We first show this for $G$ a torus $T^{c}=\left(\mathbb{C}^{*}\right)^{r}$. A $T^{c}$-action on a vector space splits it into a sum of weight spaces $W_{m}, m \in \mathfrak{t}^{*}$, on which $\exp (v) \in$ $T^{c}, v \in \mathfrak{t}^{c}$, acts as the character $\exp (i\langle m, v\rangle)$.

Given any vector $\tilde{x}$, we let $\Delta_{\tilde{x}} \subset \mathfrak{t}^{*}$ denote the convex hull of only those weights $m$ in whose weight spaces $\tilde{x}$ has nonzero components (i.e. the projection of $\tilde{x}$ to $W_{m}$ is nonzero). Any 1-PS corresponds to an integral vector $v \in \mathfrak{t}$ and so a hyperplane $H_{v} \leq \mathfrak{t}^{*}$. The points of $\Delta_{\tilde{x}}$ on the negative side of this hyperplane correspond to negative weights in whose weight space $\tilde{x}$ has a nonzero component, so their existence implies that $\lambda . \tilde{x} \rightarrow \infty$ as $\lambda \rightarrow 0$ under this 1-PS, as in (3.13). Similarly the existence of points in $\Delta_{\tilde{x}}$ on the positive side of the hyperplane prove that $\lambda . \tilde{x} \rightarrow \infty$ as $\lambda \rightarrow \infty$.

Thus $\mathbb{C}^{*} . \tilde{x}$ is closed, and $\tilde{x}$ is stable for this 1-PS, if and only if its hyperplane $H_{v} \leq \mathfrak{t}^{*}$ cuts $\Delta_{\tilde{x}}$ through its interior. Applying this to all integral points $v \in \mathfrak{t}$ (including those whose hyperplanes $H_{v}$ are parallel to the faces of $\Delta_{\tilde{x}}$ ) gives the first part of the following result, which was explained to me by Gábor Székelyhidi [Sz2].

THEOREM 4.16. The point $\tilde{x}$ is stable for every $1-P S$ if and only if $0 \in \mathfrak{t}^{*}$ is in the interior of $\Delta_{\tilde{x}}$, if and only if $\tilde{x}$ is stable for $T^{c}$.

- $=$ origin $\in \mathfrak{t}^{*}$

$\times$ weight $m \in \mathfrak{t}^{*}$

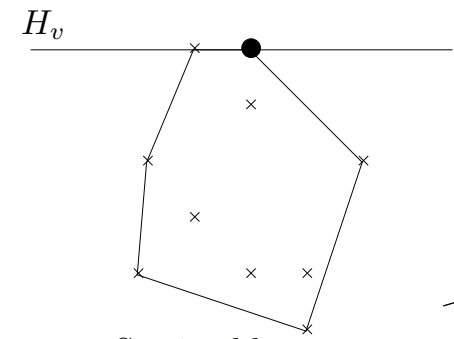

Semistable

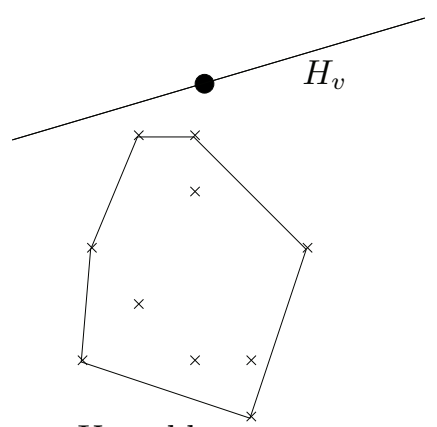

Unstable

For the second result we cover the whole of $T^{c} / T$ (where $T \leq T^{c}$ is the maximal compact subgroup $\left.T \cong\left(S^{1}\right)^{r}\right)$ by going in nonrational directions $v \in \mathfrak{t}$ too. And if the origin is in the interior, any such $v$ has negative pairing with at least one of the weights, so the associated orbit (of an analytic $\mathbb{C}$ subgroup of $T^{c}$, if $v$ is irrational) will go to infinity as we move along $v$. In fact the log-norm function will be proper with a growth that can be bounded 
below by the minimal $|\langle m, v\rangle|$. Thus the functional will be proper on all of $T^{c} / T$, and we can deduce the Hilbert-Mumford criterion.

Perhaps an easier proof, using the full Kempf-Ness theorem, comes from observing that the interior of $\Delta_{\tilde{x}}$ is the moment polytope of the orbit - the image of $T^{c} \tilde{x}$ under the moment map - so the moment map has a zero in this orbit if and only if the origin is in the interior.

But the first proof illustrates the key point, which is that the faces of $\Delta_{\tilde{x}}$ are rational - parallel to hyperplanes $H_{v}$. So if there is an irrational $v \in \mathfrak{t}$ that destabilises (has weight $\geq 0$ ) then since it cannot be contained in a face there is in fact a rational $v$, giving rise to a 1-PS, with the same property. So we can avoid the situation of a sequence of stable 1-PSs of negative weight converging to an irrational "semistable" direction of weight zero lying in a face, making the $T^{c}$-orbit non-proper but without a 1-PS or rational direction to detect it.

For an arbitrary group $G$, we can try to reduce to the torus case by dividing $G$ by $K$ on both the left and right instead of considering just $G / K$. That is, by spectral theory we can write $G=K T^{c} K$ for any maximal torus $T^{c} \leq G$; then since the norm functional is invariant under the left hand action of $K$ we are left with proving its properness on a compact family of $T^{c}$-actions - the conjugates of the original action by all $k \in K$. The result is then basically routine, the point being that in a compact family of polytopes each containing the origin in its interior, the distance of the origin to the boundary is bounded below by some $\epsilon>0$.

As an application of Theorem 4.16, we can strengthen (3.12) to recover standard results [GIT, Mu] about which hypersurfaces of degree $d$ in $\mathbb{P}^{n}$ are stable. Namely, forming the Newton polygon of degree $d$ homogeneous polynomials in $(n+1)$ variables, a hypersurface $(f=0)$ defines a subset of integral points of this polytope - those that appear in $f$ with nonzero coefficient. Then $(f=0)$ is semistable (or stable) if and only if these points do not lie to one side of (or strictly to one side of) any hyperplane through the centre of the Newton polytope.

\section{Moduli of polarised algebraic varieties $(X, L)$}

The GIT problem. This section is unnecessarily technical, and the squeamish reader can skip it once it is clear why forming moduli of algebraic varieties should be a GIT problem.

Suppose we want to form a moduli space of polarised algebraic varieties $[\mathbf{M u}$. The polarisation allows us to embed $X$ into a projective space

$$
X \hookrightarrow \mathbb{P}\left(H^{0}\left(X, L^{r}\right)^{*}\right), \quad r \gg 0 .
$$

In fact for $X$ smooth, a theorem of Matsusaka tells us that $r$ can be chosen uniformly amongst all $(X, L)$ with the same Hilbert polynomial $\mathcal{P}(r)=$ $\chi\left(X, L^{r}\right)$. Moreover we can also assume that all higher cohomology groups $H^{\geq 1}\left(X, L^{r}\right)$ vanish so that $H^{0}\left(X, L^{r}\right)$ has dimension $\mathcal{P}(r)$, and that any two 
$\left(X_{i}, L_{i}\right)$ are isomorphic if and only if their embeddings $X_{i} \hookrightarrow \mathbb{P}^{N}, N+1=$ $\mathcal{P}(r)$, differ by a projective linear map.

Picking an isomorphism

$$
H^{0}\left(X, L^{r}\right) \cong \mathbb{C}^{N+1}
$$

$(X, L)$ defines a point in the Hilbert scheme of subvarieties (in fact subschemes) of $\mathbb{P}^{N}$. This moduli space is easy to construct; for instance as a subscheme of a Grassmannian of subspaces of $S^{k}\left(\mathbb{C}^{N+1}\right)^{*} ; X \subset \mathbb{P}^{N}$ corresponding to the subspace $H^{0}\left(\mathbb{P}^{N}, \mathscr{I}_{X}(k)\right)<H^{0}\left(\mathbb{P}^{N}, \mathcal{O}(k)\right)=S^{k}\left(\mathbb{C}^{N+1}\right)^{*}$ of degree $k$ polynomials vanishing on $X$. The natural Plücker line bundle then pulls back to give an anti-ample line bundle on Hilb whose fibre at a point $(X, L)$ is

$$
\Lambda^{\max } H^{0}\left(X, L^{r k}\right)^{*} \otimes \Lambda^{\max } S^{k} H^{0}\left(X, L^{r}\right)
$$

Then we must divide out the choice of isomorphism (5.1), i.e., take the GIT quotient of Hilb by $S L(N+1, \mathbb{C})$.

So by abstract GIT, any choice of $S L(N+1, \mathbb{C}$ )-equivariant (anti-)ample line bundle on Hilb gives rise to a notion of stability for $(X, L)$. There are many such; we describe some of those whose associated weights can all be characterised in terms of weights on the line (5.2).

The Hilbert-Mumford criterion requires us to consider $\mathbb{C}^{*}<S L(N+$ $1, \mathbb{C})$ orbits of $X \subset \mathbb{P}^{N}$. This gives rise to a $\mathbb{C}^{*}$-equivariant flat family, or test configuration, $(\mathscr{X}, \mathcal{L}) \rightarrow \mathbb{C}$.

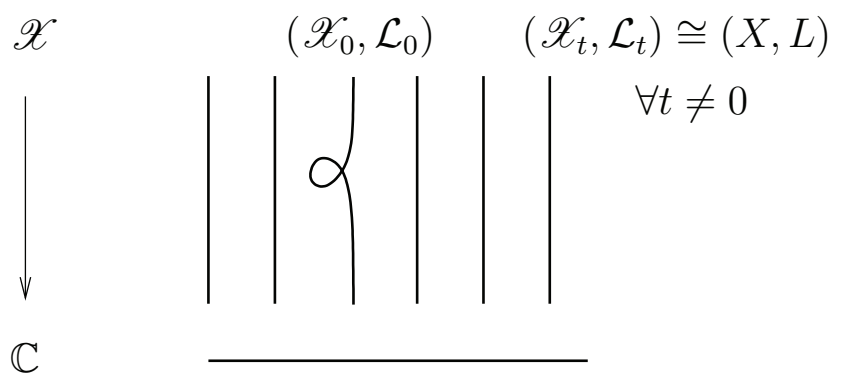

The weight $w_{r, k}$ of the $\mathbb{C}^{*}$-action on $(5.2)$ is

$$
w_{r, k}=a_{n+1}(r) k^{n+1}+a_{n}(r) k^{n}+\ldots,
$$

where

$$
a_{i}(r)=a_{i n} r^{n}+a_{i, n-1} r^{n-1}+\ldots
$$

Then doing GIT on Hilb with the line (5.2), Mumford's Chow line, or Tian's $\mathrm{CM}$ line, gives rise to Hilbert-Mumford criteria that $\mathbb{C}^{*}<S L(N+1, \mathbb{C})$ destabilises $(X, L)$ if $w_{r, k} \succ 0$ in the following senses:

- $H M(r)$-unstable: $w_{r, k}>0$ for all $k \gg 0$.

- Asymptotically HM-unstable: for all $r \gg 0, w_{r, k}>0$ for all $k \gg 0$.

- Chow(r)-unstable: leading $k^{n+1}$-coefficient $a_{n+1}(r)>0$.

- Asymptotically Chow unstable: $a_{n+1}(r)>0$ for $r \gg 0$. 
- K-unstable: leading coefficient $a_{n+1, n}>0$.

To make "if" into "iff" requires a few technicalities on the size of $r$; see [RT1]. In particular K-stability, which is Donaldson's refinement of Tian's original notion, requires one to pick a test configuration first, and then choose $r \gg 0$. The coefficient $a_{n+1, n}$ is Donaldson's version of the Futaki invariant of the $\mathbb{C}^{*}$-action on $\left(\mathscr{X}_{0}, L\right)$; see $(5.20)$.

There are also notions semistability and polystability in all of these cases; both defined by nonstrict inequalities, the latter requiring also that whenever the inequality is an equality, the test configuration should arise from an automorphism of $(X, L)$, i.e., it should be isomorphic as a scheme to the product $X \times \mathbb{C}$, but with a nontrivial $\mathbb{C}^{*}$-action.

In particular we have the following implications (see [RT1], where our $a_{i}$ are denoted $-e_{i}$ ):

Asymptotically Chow stable $\Rightarrow$ Asymptotically Hilbert stable $\Rightarrow$ Asymptotically Hilbert semistable $\Rightarrow$ Asymptotically Chow semistable $\Rightarrow$ Ksemistable.

The increasing number of test configurations that have to be tested as $r \rightarrow \infty$ currently prevents one from proving that K-stability implies asymptotic Chow stability.

The moment map problem. Fix a metric on $\mathbb{C}^{N+1}$ and so $g_{F S}$ on $\mathbb{P}^{N}$ and an induced hermitian metric on $\mathcal{O}(-1)$. This induces the symplectic form $\omega_{F S}$ on a smooth $X \subset \mathbb{P}^{N}$. This induces a natural symplectic, in fact Kähler, structure on (any smooth subset of smooth points of) Hilb:

$$
\Omega\left(v_{1}, v_{2}\right):=\int_{X} \omega_{F S}\left(v_{1}, v_{2}\right) \frac{\omega_{F S}^{n}}{n !},
$$

where the $v_{i}$ are the normal components of holomorphic vector fields along $X \subset \mathbb{P}^{N}$. This is also (a multiple of) the first Chern class of a natural line bundle on Hilb coming from the "Deligne pairing" of $\mathcal{O}_{X}(1)$ with itself $(n+1)$-times $[\mathbf{Z h}]$.

Let $m: \mathbb{P}^{N} \hookrightarrow \mathfrak{s} u(N+1)^{*}$ denote the usual moment map (4.7).

Then [Do3], just as for a finite number of points in $\mathbb{P}^{1}(4.12)$, the moment map for $S U(N+1) \curvearrowright($ Hilb,$\Omega)$ takes $X \subset \mathbb{P}^{N}$ to a multiple of its centre of mass in $\mathfrak{s} u(N+1)^{*}$ :

$$
\mu(X)=\int_{X} m \frac{\omega_{F S}^{n}}{n !} \in \mathfrak{s} u(N+1)^{*} .
$$

So zeros of moment map correspond to balanced varieties $X \subset \mathbb{P}^{N}$.

The fact that Hilb is not smooth means there are complications in applying the Kempf-Ness theorem directly, but nonetheless the following is an essentially finite dimensional result. It was first proved by Zhang $[\mathbf{Z h}]$, and then rediscovered and reproved in different forms by Luo, Paul, Wang and Phong-Sturm. 
TheOREM 5.6. $X \subset \mathbb{P}^{N}$ can be balanced by an element of $S L(N+$ $1, \mathbb{C}) \Longleftrightarrow X$ is Chow polystable.

The balanced condition can be re-expressed as follows. The metric on $\mathcal{O}_{X}(1)=L^{r}$ is the quotient metric induced from that on $H^{0}\left(\mathcal{O}_{X}(1)\right)=$ $H^{0}\left(X, L^{r}\right)$ by the surjection of vector bundles

$$
\underline{H^{0}\left(\mathcal{O}_{X}(1)\right)} \rightarrow \mathcal{O}_{X}(1) \rightarrow 0
$$

on $X$. So picking an orthonormal basis $\sigma_{i} \in H^{0}\left(X, L^{r}\right)$, we have the identity

$$
\sum_{i}\left|\sigma_{i}(x)\right|^{2} \equiv 1
$$

on $X$. (More generally, given an orthonormal basis $e_{i}$ of an inner product space $V$ and a surjection $V \stackrel{\pi}{\rightarrow} W$, we have the identity $\sum_{i}\left|\pi\left(e_{i}\right)\right|^{2}=\operatorname{dim} W$ in the induced metric on $W$. Given any basis $\sigma_{i}$ of $H^{0}\left(L^{r}\right)$ the above expression (5.7) is the pointwise ratio of the given metric on $L^{r}$ and the FubiniStudy metric on $L^{r}$ induced by embedding in $H^{0}\left(L^{r}\right)^{*}$ and pulling back the metric gotten by declaring the $\sigma_{i}$ to be orthonormal. This is constant if and only if the metric on $\left(X, L^{r}\right)$ really is such a Fubini-Study metric.)

But then in these coordinates, the moment map (5.5) constructed using (4.7), takes $X$ to the matrix with $(i j)$ th entry

$$
i\left(\int_{X} \sigma_{i}(x) \sigma_{j}(x)^{*} \frac{\omega_{F S}^{n}}{n !}-\frac{1}{N+1} \delta_{i j}\right) \in \mathfrak{s} u(N+1) .
$$

Thus the balanced condition is equivalent to the $\sigma_{i}$ being orthonormal (up to a constant scale) in the induced $L^{2}$-metric on $H^{0}\left(\mathcal{O}_{X}(1)\right)$. That is, up to scale, the original metric on $\mathbb{C}^{N+1} \cong H^{0}\left(\mathcal{O}_{X}(1)\right)=H^{0}\left(X, L^{r}\right)$ equals the $L^{2}$-metric given by integration against $\left.g_{F S}\right|_{X}$. By (5.7) this is equivalent to

$$
\sum_{i}\left|s_{i}(x)\right|^{2} \equiv \text { const, }
$$

where the $s_{i}$ are now an orthonormal basis with respect to the $L^{2}$-metric on $H^{0}\left(X, L^{r}\right)$ (rather than the original metric). A final way of saying this is that starting with a metric on $\mathbb{C}^{N+1}$ we can induce another by first inducing the Fubini-Study metric on $X \subset \mathbb{P}^{N}$ and the hermitian metric on $\mathcal{O}_{X}(-1)$, and then using this to give, by integration, an $L^{2}$-metric on $\mathbb{C}^{N+1}=H^{0}\left(\mathcal{O}_{X}(1)\right)$. Balanced metrics are then the fixed points of this operator.

Asymptotics as $r \rightarrow \infty$. Fix a metric on $(X, L)$ (e.g., by picking a metric on $H^{0}(L)$ and then inducing the Fubini-Study metric on $X \subset$ $\mathbb{P}\left(H^{0}\left(L^{*}\right)\right)$ and $\left.L=\left.\mathcal{O}(1)\right|_{X}\right)$. This then induces one on $L^{r}$ for all $r$, and so $L^{2}$-metrics on $H^{0}\left(X, L^{r}\right)$ for all $r$.

Picking an $L^{2}$-orthonormal basis $s_{i} \in H^{0}\left(X, L^{r}\right)$, we can then define, for each $r$, the Bergman kernel

$$
B_{r}\left(x_{1}, x_{2}\right)=\sum_{i} s_{i}\left(x_{1}\right) \otimes s_{i}\left(x_{2}\right)^{*}
$$


on $X \times X$. This is the integral kernel for the $L^{2}$-orthogonal projection of $C^{\infty}$ sections of $L^{r}$ onto holomorphic sections. Restricting to the diagonal gives

$$
B_{r}(x):=\sum_{i}\left|s_{i}(x)\right|^{2},
$$

so the balanced condition (5.9) is equivalent to $B_{r}$ (5.11) being constant on $X$.

This expresses the finite dimensional balanced condition (a condition for a metric on $H^{0}\left(X, L^{r}\right)$ ) as a pointwise condition for a metric on $(X, L)$ (a fact that will be explained later via Donaldson's double quotient construction) and we can look at the asymptotics of the "density of states" function $B_{r}(x)$ as $r \rightarrow \infty$ and expect it to only depend on local differential-geometric data. This is because, as is well known to quantum physicists and is made precise in [ $\mathbf{T i 3}]$, one can form a basis of sections of $H^{0}\left(L^{r}\right)$ whose norms are approximately peaked Gaussians concentrated in balls of radius const $/ \sqrt{r}$ and so volume const $/ r^{n}$. (These are the coherent states of geometric quantisation; under the metric isomorphism $H^{0}\left(L^{r}\right) \cong H^{0}\left(L^{r}\right)^{*}$ they correspond to evaluating sections at points - the centres of the peaks.) The relationship between the volume of small balls about $x \in X$ and the scalar curvature $s(x)$ at $x$ means that as $r \rightarrow \infty$ the number of peaked sections that can be packed into a fixed ball of volume $\epsilon$ about $x$ is $\sim \epsilon\left(r^{n}+\frac{n}{2} s(x) r^{n-1}+\ldots\right)$. Globally this gives rise to

$$
\operatorname{vol}(X) r^{n}+\frac{n}{2} \int_{X} s \frac{\omega^{n}}{n !} r^{n-1}+O\left(r^{n-2}\right)=\frac{L^{n}}{n !} r^{n}-\frac{K_{X} \cdot L^{n-1}}{2(n-1) !} r^{n-1}+O\left(r^{n-2}\right)
$$

sections - approximating the Riemann-Roch formula.

In fact, as $r \rightarrow \infty(\Rightarrow N \rightarrow \infty) B_{r}(x)$ has an asymptotic expansion (Tian, Zelditch, Catlin, W.-D. Ruan, Z. Lu)

$$
B_{r}(x) \sim r^{n}+\frac{1}{2 \pi} s(x) r^{n-1}+O\left(r^{n-2}\right) .
$$

More precisely, $\left\|B_{r}(x)-r^{n}+\frac{1}{2 \pi} s(x) r^{n-1}\right\|_{C^{\alpha}} \leq C r^{n-2}$ for $\alpha \geq 0$, where the constant $C$ depends on both $\alpha$ and the metric - it can only be taken to be uniform for metrics in a compact subset of the space of metrics.

Roughly speaking then, balanced metrics should tend towards cscK metrics with $[\omega]=\left[c_{1}(L)\right]$. What we have seen so far should motivate the following results.

THEOREM 5.13. If $(X, L)$ admits a cscK metric in $\left[c_{1}(L)\right]$ and has finite automorphism group then $\left(X, L^{r}\right)$ can be balanced in $\mathbb{P}\left(H^{0}\left(X, L^{r}\right)^{*}\right)$ for $r \gg$ 0. Thus it is Chow stable, and so K-semistable.

The metrics given by $r^{-1}$ times by the pull backs of the balanced metrics converge to the cscK metric.

Conversely if $\left(X, L^{r}\right) \subset \mathbb{P}^{N(r)}$ is balanced for $r \gg 0$ and the resulting $\omega_{F S, r}$ are convergent, then the limit metric is cscK. 
Finally, cscK metrics compatible with a fixed complex structure are unique up to holomorphic automorphisms of $X$.

This result is due to Donaldson [Do3]; we will discuss the proof of the balanced result in a later section. Tian had previously proved Ksemistability for KE metrics [Ti4], and a related convergence result for sequences of Fubini-Study metrics [Ti3], following a suggestion of Yau [Y2]. Using [Do3] Mabuchi proved that cscK manifolds with automorphisms are Chow polystable if the automorphisms satisfy a certain stability condition [Mb2]. Donaldson [Do6] then showed that $\operatorname{cscK} \Rightarrow \mathrm{K}$-semistable without any condition on automorphisms.

Uniqueness was originally proved by Bando-Mabuchi $[\mathbf{B M}]$ for KE metrics, by Chen $[\mathbf{C h}]$ for cscK metrics when $c_{1} \leq 0$, then by Donaldson in the general cscK case with finite automorphisms. Again the finite automorphisms condition was relaxed by Mabuchi, and, in the more general setting of extremal metrics and Kähler non-projective metrics, Chen-Tian [CT].

When our polarisation $L$ is a power of the canonical bundle $K_{X}$, then cscK metrics are in fact KE: those with Ricci form (the induced curvature of $K_{X}^{-1}$ ) a constant multiple of the Kähler form. It is clear that KE metrics are cscK; the converse follows from the calculation

$$
\Delta \operatorname{Ric}=-i \partial \bar{\partial} \Lambda \text { Ric }=-i \partial \bar{\partial} s .
$$

Here $\Lambda$ is the adjoint of $\omega \wedge$, and we use the fact that Ric is a closed real $(1,1)$-form, so it is $\partial$ - and $\bar{\partial}$-closed. $s=\Lambda$ Ric is the scalar curvature. So for $s$ a constant, Ric is harmonic, as is $\omega$. But, after scaling, they represent the same cohomology class, and so are identically equal.

KE metrics were first proved to exist on compact Kähler manifolds with positive canonical bundle by Aubin $[\mathbf{A u}]$ and Yau [Y1], and with trivial canonical bundle by Yau [Y1]. It was Calabi [Ca] who initiated the study of cscK and extremal metrics: those which extremise the Calabi functional $\int_{X} s^{2} \omega^{n}$ over cohomologous Kähler forms; they are the metrics with $\nabla s$ a holomorphic vector field. Apart from Aubin and Yau's (nonconstructive) results, there are few compact examples of cscK or KE metrics. Siu [S], Tian and Nadel $[\mathbf{N a}]$ found examples with symmetry, Tian showed Fano surfaces with reductive automorphism groups admit KE metrics [Ti2], Burns-de Bartolomeis $[\mathbf{B d B}]$ and Hong $[\mathbf{H o}]$ gave constructions of cscK metrics on certain projective bundles over cscK bases, and there are constructions for blow ups of these $[\mathbf{A P}, \mathbf{L B}, \mathbf{R S}]$ and smooth fibrations of cscK manifolds $[\mathbf{F i}]$. Bourguignon $[\mathbf{B o}]$ and Biquard $[\mathbf{B i}]$ have given excellent surveys of $\mathrm{KE}$ and $\operatorname{cscK}$ metrics respectively.

An example - blow ups of cscK manifolds. The results of [AP] give a beautiful illustration of the theory described here and the link between cscK and balanced metrics. Arezzo and Pacard consider a cscK manifold 
$(X, \omega)$ and its blow up in some points $p_{i}$,

$$
\begin{aligned}
\pi: \widehat{X} & \longrightarrow X \\
E_{i} & \rightarrow p_{i} .
\end{aligned}
$$

It is proved that there is a cscK metric in the class $\pi^{*}[\omega]-\epsilon \sum_{i} m_{i}\left[E_{i}\right]$ for $\epsilon, m_{i}>0$ and $\epsilon$ sufficiently small, if the $m_{i} p_{i}$ satisfy two conditions with respect to $\operatorname{Aut}(X, \omega)$. Arezzo and Michael Singer observed that one of these conditions could be rewritten as a balanced condition. Namely there is a moment map $X \stackrel{\mu_{a}}{\longrightarrow} \mathfrak{h a m}(X, J, \omega)^{*}$ for the action of the hamiltonian isometry group of $X$, and the conditions are that

$$
\sum_{i} m_{i} \mu_{a}\left(p_{i}\right)=0, \text { and the } \mu_{a}\left(p_{i}\right) \text { generate } \mathfrak{h a m}(X, J, \omega)^{*} .
$$

We can interpret this in the projective case, where $\mathfrak{h a m}(X, J, \omega)$ becomes $\mathfrak{a u t}(X, L)$, as follows. Taking $\epsilon$ very small is equivalent to replacing the polarisation by a very large power $r \gg 0$, whereupon the $\operatorname{cscK}$ condition approximates the balanced condition (5.12) (for what follows we only need that the approximation is valid for the linearisation of the equations as $r \rightarrow \infty)$. Then morally, in replacing $\left(X, L^{r}\right)$ by $\left(\widehat{X}, \pi^{*} L^{r}\left(-\sum m_{i} E_{i}\right)\right)$ we are perturbing a balanced $X \subset \mathbb{P}^{N}=\mathbb{P}\left(H^{0}\left(L^{r}\right)^{*}\right)$ only a little bit and so end up with a manifold that is nearly balanced. Slightly more precisely, set $I=H^{0}\left(L^{r} \otimes \mathscr{I}_{\cup_{i} m_{i} p_{i}}\right)$ and split the exact sequence

$$
0 \longrightarrow H^{0}\left(L^{r} \otimes \mathscr{I}_{\cup_{i} m_{i} p_{i}}\right) \longrightarrow H^{0}\left(L^{r}\right) \stackrel{\longrightarrow--}{\longrightarrow} \bigoplus_{i} \mathbb{C}_{p_{i}}^{m_{i}} \longrightarrow 0
$$

by picking peaked approximately Gaussian sections of $L^{r}$ on $X$ at the $p_{i}$, as in our discussion of (5.12). Away from the $p_{i}$, therefore, points in the image of $X \hookrightarrow \mathbb{P}\left(H^{0}\left(L^{r}\right)^{*}\right)$ almost annihilate this $\bigoplus_{i} \mathbb{C}_{p_{i}}^{m_{i}}$, i.e. they lie very close to $\mathbb{P}\left(I^{*}\right)$, as in the following diagram.

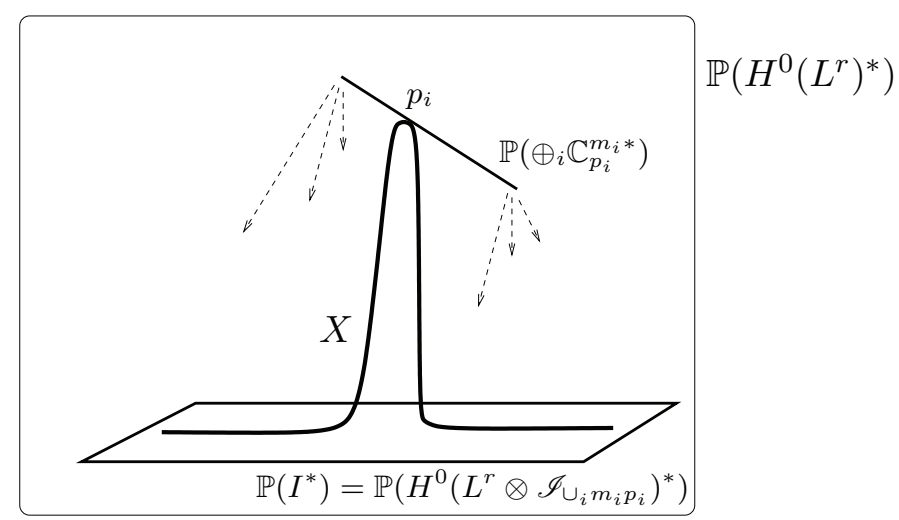

The dashed arrows denote the rational map $\mathbb{P}\left(H^{0}\left(L^{r}\right)^{*}\right)-->\mathbb{P}\left(I^{*}\right)$ blowing up $\mathbb{P}\left(\bigoplus_{i} \mathbb{C}_{p_{i}}^{*}\right)$; on restriction to $X$ this blows up the $p_{i}$ and embeds the result in $\mathbb{P}\left(I^{*}\right)$. 
So away from the $p_{i}$, the moment map $\mathbb{P}^{N} \rightarrow \mathfrak{s} u(N+1)^{*}$ of (4.7), projected to $\mathfrak{s} u(I)^{*}$, is very close (as $r \rightarrow \infty$ ) to the rational projection to $\mathbb{P}\left(I^{*}\right)$ followed by the moment map $\mathbb{P}\left(I^{*}\right) \rightarrow \mathfrak{s} u(I)^{*}$.

Since the exceptional divisors are small, we can integrate over $X$ (or its blow up in the $p_{i}$ ) to find that the centre of mass in $\mathfrak{s} u(I)^{*}$ is close to the projection of that in $\mathfrak{s} u(N+1)^{*}$. But this is zero, so $\widehat{X}$ is close to balanced, as claimed.

Now the exact sequence expressing the derivative $D$ of the $S U(N+1)$ action on the Hilbert scheme of $\mathbb{P}\left(H^{0}\left(X, L^{r}\right)^{*}\right) \supset X$,

$$
0 \rightarrow \mathfrak{a u t}\left(X, L^{r}\right) \rightarrow \mathfrak{s} u(N+1) \stackrel{D}{\longrightarrow} T \text { Hilb } \cong T^{*} \text { Hilb }
$$

(with the last isomorphism induced by the symplectic form), has dual

$$
0 \leftarrow \mathfrak{a u t}\left(X, L^{r}\right)^{*} \leftarrow \mathfrak{s} u(N+1)^{*} \stackrel{d \mu}{\longleftarrow} T \text { Hilb }
$$

by the definition of the moment map $\mu=\int_{X} m \omega_{F S} / n !(5.5)$.

If the automorphism group of $\left(X, L^{r}\right)$ is finite (so the condition (5.14) is vacuous) then $D$ is injective and its adjoint $d \mu$ is onto. So we expect to be able to move a little in the orbit to move back to a balanced metric with $\mu=0$ to correct the perturbation introduced by the $p_{i}$. This of course involves some estimates, which is what $[\mathbf{A P}]$ work out for the cscK problem, to show that for $\mathfrak{a} \mathfrak{u t}=0$ there is always a cscK metric on the blow up.

When the automorphism group is nontrivial this map $d \mu$ is not onto, so we must ensure that on perturbing as above we end up inside its image to apply the same argument. That is, by (5.15), the image of the moment map in $\mathfrak{a u t}\left(X, L^{r}\right)^{*}$ should be zero. Since the moment map is the centre of mass, and since we have added masses $m_{i}$ at the exceptional divisors $E_{i}$ lying over $p_{i}$, we must ensure that, to first order, the $\cup_{i} m_{i} p_{i}$ should be balanced in $\mathfrak{a u t}\left(X, L^{r}\right)^{*}$.

This recovers (5.14) as the necessary linearised condition. The second condition is a nondegeneracy condition that allows one to perturb the metric on and around the exceptional divisors to move the moment map enough to solve the equation to higher orders.

As pointed out by Donaldson, Hong's results $[\mathbf{H o}]$ on when a cscK metric exists on the projectivisation of a HYM bundle over a cscK base involves a similar moment map condition for the action of the automorphism group of the base on the moduli of vector bundles.

These examples illustrate a general principle about moment map problems: that transverse (regular) points of $\mu^{-1}(0)$ have no automorphisms, whereas for nontransverse points $x$ the cokernel of $d \mu$ is canonically $\left(\mathfrak{g}^{x}\right)^{*}$, the dual of the Lie algebra of the stabiliser subgroup of the point $x \in X$. Thus when one perturbs a solution $x$ of $\mu=0$ with stabiliser subgroup $G^{x}<G$, the obstruction to extending a first order deformation lies in $\left(\mathfrak{g}^{x}\right)^{*}$, and is nothing but the derivative of the moment map of the action of $G^{x}<G$. 
This follows from the exact sequence

$$
T_{x} X \stackrel{d \mu}{\longrightarrow} \mathfrak{g}^{*} \rightarrow\left(\mathfrak{g}^{x}\right)^{*} \rightarrow 0,
$$

the dual of

$$
0 \rightarrow \mathfrak{g}^{x} \rightarrow \mathfrak{g} \rightarrow T_{x}^{*} X,
$$

with the last map the composition of the $\mathfrak{g}$-action on $T_{x} X$ and contraction with the symplectic form (cf. (5.15)).

The infinite dimensional setup. Instead of letting the dimension $N$ of our quotient problem go to infinity, Donaldson [Do1] also gave a purely infinite dimensional formal symplectic quotient formulation.

The group of Hamiltonian diffeomorphisms acts on $(X, \omega)$ and so on the space of complex structures which make $(X, \omega)$ Kähler:

$\operatorname{Ham}(X, \omega) \curvearrowright \mathcal{J}:=\{\omega$-compatible complex structures on $X\}$.

Acting by pullback, the infinitesimal action of a hamiltonian $h$, with hamiltonian vector field $X_{h}$, on a complex structure $J$ is $\mathcal{L}_{X_{h}} J$. At the Lie algebra level this can be complexified so that $i h$ acts as

$$
J \mathcal{L}_{X_{h}} J=\mathcal{L}_{J X_{h}} J=\mathcal{L}_{X_{i h}} J,
$$

by the integrability of $J$. Thus it acts through the vector field

$$
X_{i h}:=J X_{h} .
$$

We note that the action of this vector field on $\omega$ is

$$
\left.\mathcal{L}_{J X_{h}} \omega=d\left(J X_{h}\right\lrcorner \omega\right)=d(J d h)=d(-i \partial h+i \bar{\partial} h)=2 i \partial \bar{\partial} h,
$$

changing $\omega$ within its cohomology class by the Kähler potential $h$ to another form compatible with $J$.

We can contract these vector fields with $\omega$ to write them as one-forms. By Hodge theory,

$$
\Omega^{1}(X)=d C^{\infty}(X) \oplus H^{1}(X, \mathbb{R}) \oplus d^{*} \Omega^{2} .
$$

The first summand corresponds to the hamiltonian vector fields, the second to symplectomorphisms modulo those which are hamiltonian, and inside the third lies $d^{*}\left(C^{\infty}(X) \omega\right)$ as those which preserve the compatibility of $\omega$ with $J$ (i.e. down which the Lie derivative of $\omega$ is of type $(1,1))$. These constitute the complexified hamiltonian action, by the Kähler identity

$$
d^{*}(h \omega)=i(\bar{\partial}-\partial) h=J d h,
$$

whose contraction with (the inverse of) $\omega$ is $J X_{h}=X_{i h}$.

So, assuming $H^{1}(X, \mathbb{R})=0$ for simplicity, integrating up this complexified Lie algebra suggests defining the complexification of $\operatorname{Ham}(X, \omega)$ to be the set of diffeomorphisms of $X$ such that the pullback of $\omega$ is compatible with $J$ (i.e., of type $(1,1))$ :

$$
\left\{f: X \rightarrow X: \exists h \in C^{\infty}(X, \mathbb{R}) \text { such that } f^{*} \omega=\omega+2 i \partial \bar{\partial} h\right\} .
$$


While this description depends on $J$, it does formally complexify $\operatorname{Ham}(X, \omega)$ : we have already seen that it has the right tangent space $C^{\infty}(X, \mathbb{R})_{0} \otimes \mathbb{C}$ at each point, and it is, crucially, contractible onto $\operatorname{Ham}(X, \omega)$ by Moser's theorem and the convexity of the space of Kähler forms.

The complexified orbits. Although (5.16) is not actually a group, its orbits on $\mathcal{J}$ (consisting of pullbacks of complex structures by the above diffeomorphisms) make perfect sense and complexify the $\operatorname{Ham}(X, \omega)$ orbits.

Since any two complex structures $J, f^{*} J$ in such an orbit differ by a diffeomorphism, we consider them isomorphic. They are both, by construction, compatible with $\omega$, but the Kähler structures $(J, \omega),\left(f^{*} J, \omega\right)$ they define need not be isomorphic as the latter is only isomorphic to $\left(J,\left(f^{-1}\right)^{*} \omega\right)$. Pulling back by the diffeomorphisms $f$ in this way (i.e., fixing $J$ and moving $\omega$ instead of the other way round) we get an exact sequence

$$
\begin{aligned}
& \operatorname{Ham}(X, \omega) \rightarrow \operatorname{Ham}^{c}(X, \omega) . J \rightarrow \\
& \left\{\text { compatible Kähler metrics on }(X, J) \text { in the } H^{2} \text { class }[\omega]\right\} .
\end{aligned}
$$

The last arrow is onto because any such $\omega^{\prime}$ is of the form $\omega+2 i \partial \bar{\partial} h$, and so diffeomorphic to $\omega$ (since by the convexity of the space of Kähler forms it is connected to $\omega$ through a family of Kähler forms $\omega+t i \partial \bar{\partial} h$ which are therefore all diffeomorphic by Moser's theorem). Thus the space of Kähler metrics on $(X, J)$ is formally of the form $G / K$. This sequence should be compared to its (more familiar) bundle analogue in (6.1).

The set-theoretic "quotient" by the complexified group (i.e., the set of complexified orbits) is therefore the set of isomorphism classes of integrable complex structures on $X$ (that are compatible with one of the symplectic forms $\left.f^{*} \omega\right)$.

Moment map = scalar curvature. The Kähler structure on $X$ induces one on $\mathcal{J}$ by integration. This is preserved by $\operatorname{Ham}(X, \omega)$, and we can ask for a moment map. Considering $C^{\infty}(X, \omega)_{0}$ (the functions of integral zero) to lie in the dual of the Lie algebra $C^{\infty}(X, \mathbb{R}) / \mathbb{R}$ by integration against $\omega^{n}$, and setting $s_{0}$ to be the topological constant $\int_{X} c_{1}(X) \cdot \omega^{n-1} / \int_{X} \omega^{n}=$ $\int s \omega^{n} / \int \omega^{n}$ (the average scalar curvature), Fujiki $[\mathbf{F} \mathbf{j}]$ and Donaldson $[\mathbf{D o 1}]$ show that

$$
\text { Moment map }=s-s_{0} \text {. }
$$

This should be no surprise, since we were looking for a function depending algebraically on the second derivatives of the metric, i.e., an invariant scalar derived from the curvature, which can only be a multiple of the scalar curvature. Thus zeros of the moment map correspond to cscK metrics. 
Norm functional = Mabuchi's K-energy. The formula (4.9) for the change in the $\log$-norm functional $M=\log \|\tilde{x}\|$ along a complexified orbit, gives the following in this infinite dimensional set-up. Moving down the orbit of $i h, h \in C^{\infty}(X, \mathbb{R})$, i.e., in the family of Kähler forms $\omega_{t}=\omega+2 i t \partial \bar{\partial} h$,

$$
\frac{d M}{d t}=m_{h},
$$

where $m_{h}=\langle m, h\rangle$ is the hamiltonian function on $\mathcal{J}$ for the element of the Lie algebra $h \in C^{\infty}(X, \mathbb{R})$. Since the moment map $m=s-s_{0}$ (5.18), $m_{h}=\int_{X}\left(s-s_{0}\right) h \omega_{t}^{n} / n !$, and

$$
M\left(\omega_{s}\right)=\int_{0}^{s} \int_{X}\left(s_{t}-s_{0}\right) h \frac{\omega_{t}^{n}}{n !} d t,
$$

where $s_{t}$ is the scalar curvature of $\omega_{t}$. This is precisely the Mabuchi functional or K-energy [Mb1], defined up to a constant (equivalent to the ambiguity in the choice of a lift of a point to the line bundle above it). It can indeed be written as the log-norm functional for a Quillen metric on a line bundle over the space of Kähler metrics; see for example [MW]. Its critical points are cscK metrics, and one expects such a metric to exist on $(X, J)$ if and only if $M$ is proper on the space of Kähler metrics on $(X, J)$ (which is the infinite dimensional analogue of $G / K$ by (5.17)).

Weight $=$ Futaki invariant. The formula (5.19) at a fixed point (e.g. the limit point of a 1-PS when this exists and is smooth), on the line over which $\mathbb{C}^{*}$ acts with weight $\rho$, is

$$
\frac{d M}{d t}=\rho=m_{h}=\int_{X}\left(s-s_{0}\right) h \frac{\omega^{n}}{n !} .
$$

Compare $(4.5,4.9,4.10)$. This is the statement that "the derivative of the Mabuchi energy is the Futaki invariant" [Mb1, DT].

The right hand side is, up to a sign, the original definition of the Futaki invariant $[\mathbf{F u}]$ for a smooth polarised manifold $(X, L)$ with a $\mathbb{C}^{*}$-action. Noting as above that it is the weight of the induced action on a line led Donaldson to give the more general definition $a_{n+1, n}$ described earlier, for an arbitrary polarised scheme $(X, L)$.

Approximation and quantisation. As Donaldson explains in [Do4], the finite dimensional problem of balanced metrics can be thought of as the quantisation of the infinite dimensional problem of cscK metrics, which emerges as the classical limit as $r, N \rightarrow \infty$.

As in quantum theory we think of the spaces of sections of the line bundle $L^{r}$ as wave functions on $X$, with a basis of Gaussian sections, peaked around points on $x$. As $r \rightarrow \infty$ these peak more, largely supported in balls of radius const $/ r$. Our $S L(N+1, \mathbb{C})$ group action moves these sections around the manifold, which may be thought of as moving quantised chunks of manifold of volume $\sim 1 / r^{n}$ around $X$ (thanks to Anton Gerasimov for this analogy). In the limit this is meant to approximate the classical limit 
of the diffeomorphisms (5.16) in the complexification of $\widetilde{\operatorname{Ham}}(X, \omega)$ moving points of the manifold around.

There is in fact a natural map $\mathfrak{s} u(N+1) \rightarrow C^{\infty}(X, \mathbb{R})$, though it is only a homomorphism of Lie algebras to leading order in $r[\mathbf{C G R}]$. A skewadjoint endomorphism $i A \in \mathfrak{s} u(N+1)$ gives an infinitesimal automorphism of $\mathbb{P}^{N}$ whose vector field $v_{A}$ is hamiltonian with respect to the Fubini-Study symplectic form. Its hamiltonian is the function (Berezin symbol)

$$
\mathbb{P}^{N} \ni x=[\tilde{x}] \mapsto \frac{\langle A \tilde{x}, \tilde{x}\rangle}{\|\tilde{x}\|^{2}}=: h_{A} .
$$

On $X,\left.h_{A}\right|_{X}$ induces a hamiltonian vector field which is the orthogonal projection of $v_{A}$ from $\left.T \mathbb{P}^{N}\right|_{X}$ to $T X$.

Using the fact that $T X$ is invariant under the complex structure $J$, and working with complexified hamiltonian vector fields (of the form $X_{h}+$ $\left.J X_{g}=: X_{h+i g}\right)$, the same working shows that the same formula defines a map from $\mathfrak{s l}(N+1, \mathbb{C})$ to the Lie algebra $C^{\infty}(X, \mathbb{C})$ of the complexification (5.16) of $\widetilde{\operatorname{Ham}}(X, \omega)$. Thus the change in metric on $X$ induced by pulling back the metric along an $\mathfrak{s l}(N+1, \mathbb{C})$ vector field in $\mathbb{P}^{N}$ is the same as that induced by pulling back along its orthogonal projection tangent to $X$. (Thanks to Gábor Székelyhidi for this observation [Sz2].)

In this way algebraic 1-PS orbits, i.e. test configurations, give rise to curves in the complexification of the $\widetilde{\operatorname{Ham}}(X, \omega)$-action on $\mathcal{J}$ which approximate 1-PS orbits. Using the description of these orbits in terms of a fixed complex structure and varying Kähler form (5.17), this simply corresponds to restricting the Fubini-Study metric of $\mathbb{P}^{N}$ to the test configuration.

To get a map back we orthogonally project the prequantisation representation $\widetilde{\operatorname{Ham}}(X, \omega) \rightarrow \operatorname{Aut}\left(\Gamma\left(L^{r}\right)\right)$ to $H^{0}\left(L^{r}\right)<\Gamma\left(L^{r}\right)$ using the Bergman kernel (5.10). That is $h \in C^{\infty}(X, \mathbb{R})$ maps to the infinitesimal automorphism $i A \in \mathfrak{s} u\left(H^{0}\left(L^{r}\right)\right)$ defined by

$$
i A(s)=\sum_{i}\left\langle\nabla_{X_{h}} s+i h s, s_{i}\right\rangle_{L^{2}} s_{i} .
$$

Again, this is not a homomorphism (except to leading order in $r$ ). The problem is that we had to use the Bergman kernel because quantisation is not a symplectic invariant (it cannot be done equivariantly with respect to symplectomorphisms or elements of $\widetilde{\operatorname{Ham}}(X, \omega))$. That is, it is not independent of choices of complex structure because the pullback of $s \in H^{0}\left(L^{r}\right)$ by $\widehat{\operatorname{Ham}}(X, \omega)$ is not in general holomorphic.

Donaldson's double quotient construction. Because of this problem Donaldson [Do3] considers pairs of a complex structure $J \in \mathcal{J}$ and a section $s \in \Gamma\left(L^{r}\right)$ which is holomorphic with respect to $J$; these are clearly acted on by $\widetilde{\operatorname{Ham}}(X, \omega)$. In fact he considers $N+1=h^{0}\left(X, L^{r}\right)$-tuples of 
sections:

$$
\mathcal{S}=\left\{\left(J,\left\{s_{i}\right\}\right) \in \mathcal{J} \times \Gamma\left(L^{r}\right)^{N+1}: \bar{\partial}_{J} s_{i}=0, i=1, \ldots, N+1\right\} .
$$

Here, as usual, $L$ has a metric and hermitian connection, and $\bar{\partial}_{J}$ is the the $(0,1)$-part of the induced connection on $L^{r}$ with respect to the complex structure $J$. Since the curvature $2 \pi r i \omega$ is compatible with $J$ (by the definition of $\mathcal{J}$ ), i.e. of type $(1,1), \bar{\partial}_{J}^{2}=0$ and $\bar{\partial}_{J}$ defines an integrable holomorphic structure on $L^{r}$ by the Newlander-Nirenberg theorem.

We now have actions of $G L(N+1, \mathbb{C})$ and $\widetilde{\operatorname{Ham}}(X, \omega)^{c}$. These commute, and both have centre $\mathbb{C}^{*}$ acting by scalars on $L$, so we can quotient by $\widetilde{\operatorname{Ham}}(X, \omega)^{c}$ and then $S L(N+1, \mathbb{C})$, or by $G L(N+1, \mathbb{C})$ and then $\operatorname{Ham}(X, \omega)^{c}$. In this way we will see how an infinite dimensional moment map problem is equivalent to a finite dimensional one.

Dividing by $G L(N+1, \mathbb{C}$ ) leaves $\mathcal{J}$ (with a fibration over it by the Grassmannian of $(N+1)$-planes in $H^{0}\left(L^{r}, \bar{\partial}_{J}\right)$, by Proposition 4.14, but for $L$ sufficiently ample $N+1=h^{0}\left(L^{r}\right)$ and this is a single point). In turn the formal complex quotient of this by $\operatorname{Ham}(X, \omega)^{c}$, discussed above, is the space of complex structures on $X$ (compatible with some symplectic structure in the diffeomorphism group orbit of $\omega$ ). Taking symplectic reductions instead we end up with cscK metrics (together with orthonormal bases of $H^{0}\left(L^{r}\right)$ modulo the unitary group - i.e. just a point). So far then, we have just reproduced what we already knew.

However, we can put a different symplectic structure $\Omega_{r}$ on $\mathcal{J}$, and one that tends to $\Omega$ as $r \rightarrow \infty$. Namely, the fact that the $s_{i}$ determine an embedding of $X$ into $\mathbb{P}\left(H^{0}\left(L^{r}\right)^{*}\right)$ for $r \gg 0$ means that the natural projection

$$
\mathcal{S} \rightarrow \Gamma(L)^{N+1}
$$

is an embedding, and we can pullback the natural $L^{2}$-symplectic form from the latter to define $\Omega_{r}$.

Now [Do3, Do4] the moment map for the $\operatorname{Ham}(X, \omega)$-action becomes

$$
\left(\frac{1}{2} \Delta+r\right) \sum_{i}\left|s_{i}(x)\right|^{2},
$$

with zeros the solutions of $\sum_{i}\left|s_{i}(x)\right|^{2}=$ constant.

If we first take the symplectic reduction by $\operatorname{Ham}(X, \omega)$ then this involves solving $\sum_{i}\left|s_{i}(x)\right|^{2}=$ constant, which we have already observed in (5.7) says that the metric on $X$ is the restriction of the Fubini-Study metric on $\mathbb{P}\left(H^{0}\left(L^{r}\right)^{*}\right) \supset X$ when we put the metric on $H^{0}\left(L^{r}\right)$ that makes the $r s_{i}$ orthonormal (the scaling arising because we have ignored the central scalar action). But since this is a Kähler metric in the same class as $\omega$, we have already observed (5.17) that we can solve this in $\operatorname{Ham}(X, \omega)^{c}$ orbit, uniquely up to the action of $\operatorname{Ham}(X, \omega)$. Next we take the reduction by $S U(N+1, \mathbb{C})$, which by (5.5) means we try to balance $X \subset \mathbb{P}\left(H^{0}\left(L^{r}\right)^{*}\right)$ in the metric in which the $r s_{i}$ are orthonormal. By Theorem 5.6 there is a solution to this 
problem in a $S L(N+1, \mathbb{C})$-orbit of $X$, unique up to $S U(N)$, if and only if $X \subset \mathbb{P}\left(H^{0}\left(L^{r}\right)^{*}\right)$ is Chow polystable.

So that gives us the finite dimensional problem of solving (5.8), (which, as observed there, is equivalent to the metric on $H^{0}\left(L^{r}\right)$ being the $L^{2}$ metric).

Taking the symplectic reduction in the opposite direction gives instead the pointwise description (5.9) of the balanced condition. Namely, first taking the reduction by $S U(N+1)$ gives us an orthonormal basis $s_{i}$ (up to an overall scale which could be removed by putting back the central $\mathbb{C}^{*}$ action) in each $S L(N+1, \mathbb{C})$ orbit, unique up to $S U(N+1)$, if and only the original $s_{i}$ were linearly independent (Proposition 4.14). Then taking the reduction by $\operatorname{Ham}(X, \omega)$ involves solving $(5.9) B_{r}(x)=$ const for the metric. So we see how solving this infinite dimensional moment map problem has been reduced to the finite dimensional balanced moment map problem.

This latter equation has the advantage that it is asymptotically close to the cscK equation (5.12). If quantisation really "worked" it would be exactly the cscK equation, and proving Donaldson's result that $\operatorname{cscK} \Rightarrow$ balanced would be trivial. Since it is only asymptotically close, Donaldson crucially uses the "failure" of quantisation to move from a cscK solution to a balanced solution, as we now describe.

CscK $\Rightarrow$ balanced. In [Do3], Donaldson proves a "quantitative" version of the Kempf-Ness theorem: if the moment map $m(x)$ at a point $x$ is small, and the action of the Lie algebra at $x$ is injective, with a sufficiently large lower bound on its smallest eigenvalue in a sufficiently large neighbourhood of $x$, then there exists a zero of $m$ close to $x$ in its complexified orbit. Flowing down the gradient of $-\|m\|^{2}$, i.e. down $J X_{m^{*}}$ (where $m^{*} \in \mathfrak{k}$ is dual to $m \in \mathfrak{k}^{*}$ under the Killing form), the conditions ensure that $X_{m^{*}}$ is sufficiently large and so $\|m\|^{2}$ decreases sufficiently fast for sufficiently long to converge to a zero of $m$.

He applies this to the $S U(N+1)$-action on the symplectic reduction of $\mathcal{S}$ by $\operatorname{Ham}(X, \omega)$. The cscK metric ensures that we are close to a balanced metric (zero of the moment map) as $r \rightarrow \infty$. Then to give a lower bound for the injectivity of the $\mathfrak{s} u(N+1)$-action it is equivalent to give a bound for the orthogonal projection of its action perpendicular to the orbits of $\operatorname{Ham}(X, \omega)$ upstairs on $\mathcal{S}$.

Donaldson shows that the projection of the action of $i A \in \mathfrak{s} u(N+1)$ onto the tangent to the $\operatorname{Ham}(X, \omega)$ orbits is just what one might expect from quantisation: it is the action of its Berezin symbol $h_{A}$ (5.21). So the normal projection we require is given by the difference in the actions of $i A$ and $h_{A}$ on $\mathcal{S}$.

It is here is where the failure of quantisation to be equivariant with respect to $\operatorname{Ham}(X, \omega)$ is used - to show that this difference is sufficiently large in some sense. Of course quantisation is invariant with respect to 
holomorphic hamiltonian vector fields, i.e., those functions satisfying

$$
\left.\mathcal{D} h:=\bar{\partial} X_{h}=\bar{\partial}(d h\lrcorner \omega^{-1}\right)=0 .
$$

[Do3] assumes that $\operatorname{Aut}(X, J)=0$, so that ker $\mathcal{D}$ is just the constants. Then the (fixed) lowest eigenvalue of $\mathcal{D}$ gives the lower bound on the difference of the actions of $i A$ and $h_{A}$. This gives the required estimates, as $r \rightarrow \infty$ and we get closer to a zero of the balanced moment map equation, to apply Donaldson's quantitative Kempf-Ness theorem.

So $S U(N+1)$ really "approximates" $\widetilde{\operatorname{Ham}}(X, \omega)$, in the sense that its finite dimensional moment map converges to the infinite dimensional one (5.12), the symplectic structures $\Omega_{r} \rightarrow \Omega$, and the natural norm functionals and weights tend to their infinite dimensional analogues (the Mabuchi functional and Futaki invariant) as $r \rightarrow \infty$; see [Do4]. Also the space of "algebraic metrics" (the restrictions of the Fubini-Study metrics $S L(N+1, \mathbb{C}) \cdot \omega_{F S}$ from $\mathbb{P}^{N}$ ) becomes dense in the space of all Kähler metrics as $r, N \rightarrow \infty$ [Ti3]. Thus the quantum picture tends to the classical one as $r \rightarrow \infty$.

The Yau-Tian-Donaldson conjecture. By analogy with the KempfNess theorem in finite dimensions (and by taking the infinite limit of Theorem 5.6) it is natural to conjecture a Hitchin-Kobayashi correspondence (the name coming from the analogy with the bundle case in the next section). That is a variety should admit a cscK metric if and only if it is polystable in a certain sense.

In fact Yau [Y3] first suggested that there should be a relationship between stability and the existence of KE metrics. Tian [Ti2] proved this for surfaces, introduced his notion of K-stability, and, building on his work with Ding $[\mathbf{D T}]$, showed it was satisfied by Kähler-Einstein manifolds [Ti4]. The definition of K-stability was generalised to more singular test configurations by Donaldson [Do5] who also showed that cscK implies K-semistability [Do3]. So it was thought that K-polystability, as defined above, should be the right notion to be equivalent to $\operatorname{cscK}$.

Recent explicit examples [ACGT] in the extremal metrics case (where there is a similar conjecture due to Székelyhidi [Sz1]) suggest that this should be strengthened to analytic K-polystability, allowing more general analytic (instead of just algebraic) test configurations. In particular one should allow the line bundle $L$ over the test configuration to be an $\mathbb{R}$-line bundle: an $\mathbb{R}$-linear combination (by tensor product) of $\mathbb{C}^{*}$-linearised line bundles. So the most likely Yau-Tian-Donaldson conjecture as things stand at the end of 2005 is the following.

Conjecture 5.22. $(X, L)$ is analytically K-polystable $\Longleftrightarrow(X, L)$ admits a cscK metric. This is unique up to the holomorphic automorphisms of $(X, L)$.

This would be the right higher dimensional generalisation of the uniformisation theorem for Riemann surfaces. 
There is very little progress on this conjecture in the $\Rightarrow$ direction except for projective bundles $[\mathbf{B d B}, \mathbf{H o}, \mathbf{R T 2}]$ and Donaldson's deep work on toric surfaces [Do5]. In the KE case there are sufficient conditions for existence given by Tian's $\alpha$-invariant [Ti1] and Nadel's multiplier ideal sheaf [Na], but no one has successfully related these to stability. Part of the problem, quite apart from the analytical difficulties, is that we do not have a good intrinsic understanding of stability for varieties - i.e., no one has successfully analysed the Hilbert-Mumford criterion for varieties.

Summarising the status of the whole theory for varieties, we have the infinite dimensional analogue of the balanced condition for points in $\mathbb{P}^{1}$ (i.e., cscK metrics) and part of the relationship to stability, but not the algebrogeometric description of stability. That is, the Hilbert-Mumford criterion, giving the analogue of the multiplicity $<n / 2$ condition for points in $\mathbb{P}^{1}$, is missing.

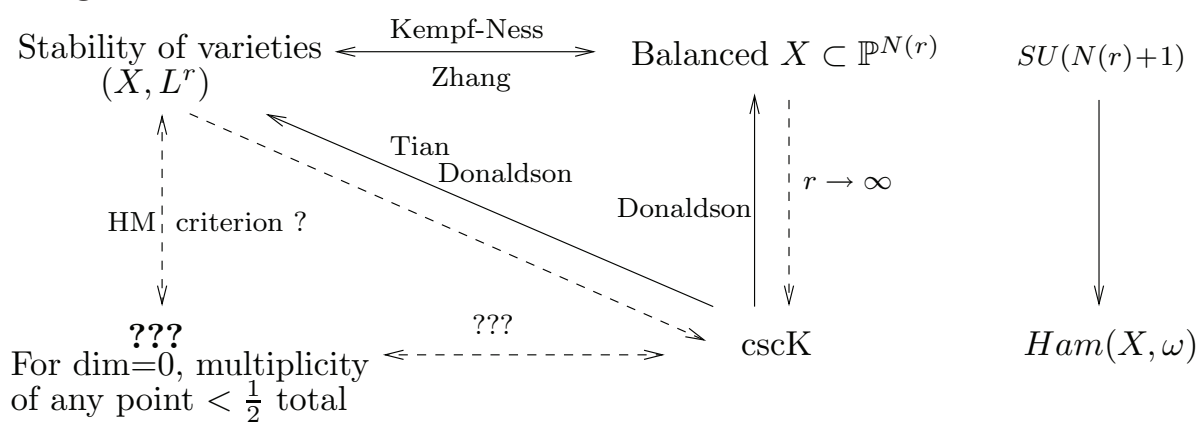

\section{Moduli of bundles over $(X, L)$}

For holomorphic bundles $E$ over a polarised algebraic variety $(X, L)$ there is a very similar story which is more-or-less completely worked out. Again there are subtleties due to different notions of stability, but for bundles for which Gieseker and slope stability coincide, for simplicity (such as those with coprime rank and degree, or bundles over curves), we have, for $r \gg 0$,

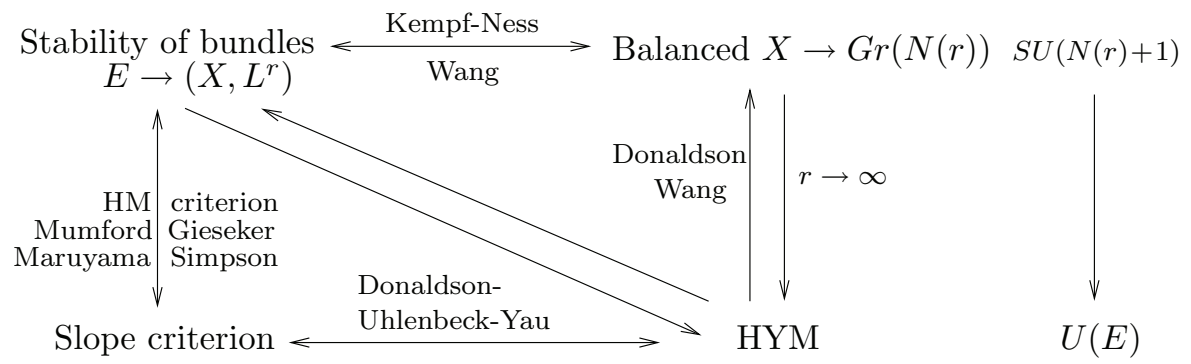

We now briefly explain this theory.

The gauge theory picture. The formal infinite dimensional picture was described by Atiyah-Bott $[\mathbf{A B}]$. Fix a compatible hermitian metric on a $C^{\infty}$-bundle $E$ and consider the gauge group $U(E)=\left\{\right.$ unitary $C^{\infty}$-maps 
$E \rightarrow E\}$ and its (genuine) complexification $G L(E)$ of all $C^{\infty}$ invertible bundle maps $E \rightarrow E$. These act on

$$
\mathcal{A}=\left\{\text { unitary connections } A \text { with } F_{A}^{0,2}=0\right\} .
$$

The $U(E)$-action is obvious; $G L(E)$ acts by pulling back the $(0,1)$-part $\bar{\partial}_{A}$ of the connection and then taking the unique Chern connection compatible with both this and the metric. The integrability condition $F_{A}^{0,2}=\bar{\partial}_{A}^{2}=0$ ensures that $\bar{\partial}_{A}$ defines a holomorphic structure on $E$. Thus any two $\bar{\partial}$ operators define isomorphic holomorphic structures on $E$ if and only if they lie in the same $G L(E)$-orbit. So the formal complex quotient of $\mathcal{A}$ by $G L(E)$ is the moduli space of holomorphic vector bundles on $X$ with topological type E. (Of course we expect to need a stability condition to form this quotient.)

Alternatively, fixing the $\bar{\partial}$-operator and pulling back the metric by $G L(E)$ gives the direct analogue of (5.17) for the complexified orbit of $\bar{\partial}_{A}$ :

$$
U(E) \rightarrow G L(E) . \bar{\partial}_{A} \rightarrow\left\{\text { compatible metrics on }\left(E, \bar{\partial}_{A}\right)\right\} .
$$

The last map is onto since $G L(E)$ acts transitively on the space of compatible hermitian metrics on $E$ (the space of metrics being $G L(E) / U(E)$ ), so a complexified orbit can be thought of as giving all compatible metrics on a fixed holomorphic bundle $\left(E, \bar{\partial}_{A}\right)$, up to the action of $U(E)$.

Fix a compatible hermitian metric on $L$, inducing a Kähler form $\omega$ on $X$. Then $\mathcal{A}$ inherits a natural Kähler structure, with symplectic form given by $\Omega(a, b)=\int_{X} \operatorname{tr}(a \wedge b) \wedge \omega^{n-1}$ for $a, b \in \Omega^{1}($ End $E)$ tangent vectors to $\mathcal{A}$. Atiyah-Bott show that $U(E) \curvearrowright \mathcal{A}$ has a moment map

$$
A \mapsto F_{A}^{1,1} \wedge \omega^{n-1}-\lambda \operatorname{id} \omega^{n} \in \Omega^{2 n}(\mathfrak{s} u(E)),
$$

thinking of the latter space as dual to $\Omega^{0}(\mathfrak{s} u(E))$ by the trace pairing and integration. Here $\lambda=2 \pi i \mu(E) / \int_{X} \omega^{n}$ is a topological constant, where

$$
\mu(E)=\frac{\int_{X} c_{1}(E) \cdot \omega^{n-1}}{\operatorname{rank} E}
$$

is the slope of $E$.

Thus zeros of the moment map are Hermitian-Yang-Mills connections; solutions of $\Lambda F_{A}^{1,1}=$ const.id. An infinite dimensional version of the Kempf-Ness theorem would be that in a polystable orbit of $G L(E)$ there should be a HYM connection (i.e. a metric whose associated Chern connection is HYM; we call this a HYM metric), unique up to the action of $U(E)$, as conjectured by Hitchin and Kobayashi.

TheOREM 6.3 (Donaldson-Uhlenbeck-Yau). E slope polystable $\Longleftrightarrow E$ admits a HYM metric. It is unique up to the automorphisms of $E$.

The notion of stability that arises here (also called Mumford stability) comes from GIT. 
The GIT picture. Suppose we wanted form an algebraic moduli space of bundles $E$ over $(X, L)$ of fixed topological type. (More generally, to get a compact moduli space, we have to consider coherent sheaves $E$ of the same Hilbert polynomial $\chi(E(r))$.) We can twist $E(r):=E \otimes L^{r}, r \gg 0$ until (a bounded subset of) the $E$ s have no higher cohomology and are generated by their holomorphic sections:

$$
0 \rightarrow \operatorname{ker} \rightarrow \underline{H^{0}(E(r))} \rightarrow E(r) \rightarrow 0 \quad \text { on } X .
$$

Fixing an isomorphism $H^{0}(E(r)) \cong \mathbb{C}^{N}, N=\chi(E(r))$, we have expressed all such $E$ s as quotients of $\mathcal{O}(-r)^{\oplus N}$. Such quotients are easily parameterised algebraically by a Quot scheme (for instance as a subset of the Grassmannian of subspaces $H^{0}(\operatorname{ker}(s)) \leq H^{0}(\mathcal{O}(s))^{\oplus N}$ given by taking sections of $(6.4)$ tensored by $L^{s}, s \gg 0$.)

So we divide by choices of the isomorphism $H^{0}(E(r)) \cong \mathbb{C}^{N}$, i.e., by $S L(N, \mathbb{C})$, to get the moduli space of (semistable) sheaves.

In this case the Hilbert-Mumford criterion can be manipulated (Mumford, Takemoto, Gieseker, Maruyama, Simpson $[\mathbf{H L}]$ ) to give an algebrogeometric understanding of stability. We describe this in a later section; the upshot is the following.

We write the Hilbert polynomial $\chi(E(r))=a_{0} r^{n}+a_{1} r^{n-1}+\ldots$, where $a_{0}=\operatorname{rank} E \int_{X} \omega^{n} / n !, \quad a_{1}=\int_{X} c_{1}(E) \cdot \omega^{n-1} /(n-1) !+\varepsilon(X)$, etc. are topological. We use its monic version, the reduced Hilbert polynomial

$$
p_{E}(r):=\frac{\chi(E(r))}{a_{0}}=r^{n}+\frac{a_{1}}{a_{0}} r^{n-1}+\ldots .
$$

Then $E$ is stable if and only if for all coherent subsheaves $F \hookrightarrow E, p_{F}(r)$ $\prec p_{E}(r)$ in the following sense (depending on the line bundle chosen on the Quot scheme):

- Gieseker stable if and only if $p_{F}(r)<p_{E}(r) \quad \forall r \gg 0$.

- Slope stable if and only if $\frac{a_{1}(F)}{a_{0}(F)}<\frac{a_{1}(E)}{a_{0}(E)}(\Longleftrightarrow \mu(F)<\mu(E))$.

Here, as before, $\mu(E)=\int_{X} c_{1}(E) \cdot \omega^{n-1} / \operatorname{rank}(E)$ is the slope of $E(6.2)$. Gieseker and slope stability coincide on curves $X$. Slope stability corresponds to taking a certain semi-ample line bundle on the Quot scheme (roughly speaking given by restricting sheaves to high degree complete intersection curves in $X$ and using the usual line bundle for moduli of bundles on the curve). GIT needs amending for this situation; so far this has been carried out only for $X$ a surface by Jun Li $[\mathbf{H L}]$.

Semistability is similar (replacing $<$ by $\leq$ ), while polystability is equivalent to semistability where the only destabilising subsheaves $F$ are direct summands of $E$. Slope polystability then turns out to be the right stability notion for the infinite dimensional quotient and HYM of Theorem 6.3.

The symplectic reduction picture. For $E$ a bundle, we can interpret the quotient $\underline{\mathbb{C}}^{N} \rightarrow E(r) \rightarrow 0$ (6.4) differently, via its classifying map $X \rightarrow$ $G r$ to the Grassmannian of quotients of $\mathbb{C}^{N}$. 
Then, much as in the varieties case, we fix compatible hermitian metrics on $L$ and $E$ inducing a Kähler form on $X$ and an $L^{2}$-metric on $\mathbb{C}^{N} \cong$ $H^{0}(E(r))$. Then there are actions of $S U(N)<S L(N, \mathbb{C})$ on $G r$, inducing a moment map $m: G r \hookrightarrow \mathfrak{s} u(N)^{*}$ and an action $S U(N) \curvearrowright \operatorname{Maps}(X, G r)$. Its moment map is the integral of (the pullback of) $m$ over $X$, so we can again talk about balanced $X \rightarrow G r$ (those with centre of mass zero in $\left.\mathfrak{s} u(N)^{*}\right)$ and asymptotics as $r, N \rightarrow \infty$.

Proving conjectures of Donaldson [Do2], Wang shows that the existence of a balanced map is equivalent to the Gieseker polystability of $E$ [Wa1]. Slope stable bundles (which are therefore Gieseker stable) admit balanced maps $X \rightarrow G r$ for $r \gg 0$ [Wa2], and pulling back the canonical quotient connection on $G r$ and taking $\lim _{r \rightarrow \infty}$ gives a conformally Hermitian-YangMills connection on $E$ (which is HYM after rescaling). (Unfortunately, this is not how the results are proved; Wang uses the Donaldson-Uhlenbeck-Yau theorem to give an a priori HYM connection which can be compared to the sequence of balanced metrics.)

\section{Slope criteria for algebraic varieties}

Slope for K-stability. So we have seen that the finite and infinite dimensional GIT and symplectic reduction pictures work for bundles, and tend to one another as $r \rightarrow \infty$. In particular the stability notion for bundles and sheaves involves only subsheaves $F<E$. So one might ask if the subvariety $\mathbb{P}(F) \subset \mathbb{P}(E)$ can destabilise the variety $\mathbb{P}(E)$. Or if, more generally, subschemes $Z \subset(X, L)$ can destabilise $(X, L)$. (Cf. length $\geq n / 2$ subschemes destabilising length- $n$ schemes in $\mathbb{P}^{1}(3.10)$.)

We need some topological data for $(X, L)$ analogous to that for bundles (6.5). Fixing $Z \subset(X, L)$, we have the Hilbert polynomial of $L=\mathcal{O}_{X}(1)$

$$
h^{0}\left(\mathcal{O}_{X}(r)\right)=a_{0} r^{n}+a_{1} r^{n-1}+\ldots,
$$

and the Hilbert-Samuel polynomial of $\mathscr{I}_{Z}($ for $x \in \mathbb{Q}$ and $r x \in \mathbb{N})$ :

$$
h^{0}\left(\mathscr{I}_{Z}^{x r}(r)\right)=a_{0}(x) r^{n}+a_{1}(x) r^{n-1}+\ldots .
$$

Then by working on the blow up of $X$ in $Z$ one can see that the $a_{i}(x)$ are polynomials in $x \in \mathbb{Q} \cap[0, \epsilon(Z)$ ) for $r \gg 0$. (More precisely, there is a constant $p$ and a polynomial which is equal to $a_{i}(x)$ for $x r>p$ or $x=0$.) Here $\epsilon(Z)$ is the Seshadri constant of $Z$, the supremum of $x$ such that $\mathscr{I}_{Z}^{x r}(r)$ is generated by global sections for $r \gg 0$.

For $X$ normal, $a_{0}(0)=a_{0}$, and $a_{1}(0)=a_{1}$. All the $a_{i}(x)$ are given by topological formulae by Riemann-Roch, for instance

$$
a_{0}=\frac{\int_{X} \omega^{n}}{n !}, \quad a_{1}=\frac{\int_{X} c_{1}(X) \omega^{n-1}}{2(n-1) !} .
$$


For any $c \leq \epsilon(Z)$, analogously to the definition of slope for bundles (6.2), we define the slope of $Z$ to be

$$
\mu_{c}\left(\mathscr{I}_{Z}\right)=\frac{\int_{0}^{c} a_{1}(x)+\frac{a_{0}^{\prime}(x)}{2} d x}{\int_{0}^{c} a_{0}(x) d x} .
$$

$Z=\emptyset$ gives

$$
\mu(X)=\frac{a_{1}}{a_{0}} .
$$

We then have the following [RT2].

Theorem 7.2. $(X, L) K$-semistable $\Rightarrow$ slope semistable: $\mu_{c}\left(\mathscr{I}_{Z}\right) \leq \mu(X)$ for all closed subschemes $Z \subset X$ and $c \leq \epsilon(Z)$.

Removing the "semi" is a little more involved. If we use the algebraic K-stability of [Do5, RT1] then we define slope stability to mean

- $\mu_{c}\left(\mathscr{I}_{Z}\right)<\mu(X) \forall c \in(0, \epsilon(Z)) \cap \mathbb{Q}$, and

- $\mu_{\epsilon(Z)}\left(\mathscr{I}_{Z}\right)<\mu(X)$ if $\epsilon(Z) \in \mathbb{Q}$ and $\mathscr{I}_{Z}^{\epsilon(Z) r}(r)$ is saturated by global sections for $r \gg 0$.

(The quickest definition of saturated [RT1] is that on the blow up $\pi: \mathrm{Bl}_{Z} X$ $\rightarrow X$ of $X$ in $Z$ with exceptional divisor $E, \pi^{*} \mathscr{I}_{Z}^{\epsilon(Z) r}(r)=\pi^{*} L^{r}(-\epsilon(Z) r)$ should be generated by global sections.) Similarly slope polystability is defined as slope stability except in the second part of the definition we allow $\mu_{\epsilon(Z)}\left(\mathscr{I}_{Z}\right)$ to equal $\mu(X)$ if on $\mathrm{Bl}_{Z \times\{0\}}(X \times \mathbb{C}), L(-\epsilon(Z) P)$ (where $P$ is the exceptional divisor) is pulled back from a contraction $\mathrm{Bl}_{Z \times\{0\}}(X \times \mathbb{C}) \rightarrow$ $X \times \mathbb{C}$ (which of course won't be the original blowup map).

If we use analytic K-stability [RT2], which is what should be relevant to the cscK problem (Conjecture 5.22), then the relevant definition of slope stability allows irrational $c$ :

- $\mu_{c}\left(\mathscr{I}_{Z}\right)<\mu(X) \forall c \in(0, \epsilon(Z))$, and

- $\mu_{\epsilon(Z)}\left(\mathscr{I}_{Z}\right)<\mu(X)$ if $\epsilon(Z) \in \mathbb{Q}$ and $\mathscr{I}_{Z}^{\epsilon(Z) r}(r)$ is saturated by global sections for $r \gg 0$.

Again slope polystability is defined in the same way except for the second condition in which we allow $\mu_{\epsilon(Z)}\left(\mathscr{I}_{Z}\right)=\mu(X)$ if on $\mathrm{Bl}_{Z \times\{0\}}(X \times$ $\mathbb{C}), L^{r}(-\epsilon(Z) r P)$ is pulled back from a contraction to $X \times \mathbb{C}$.

Then the analogue of Theorem 7.2, for the either notion of K-stability, is the following [RT1].

Theorem 7.3. $(X, L) K$-(poly)stable $\Rightarrow$ slope (poly)stable.

As a corollary of Theorem 7.2 and the results of Donaldson and ChenTian mentioned in Theorem 5.13 we find the following.

Corollary 7.4. If $\mu_{c}\left(\mathscr{I}_{Z}\right)>\mu(X)$ then $X$ does not admit a cscK metric in the class of $c_{1}(L)$.

We give some examples. 
- If $F<E$ is a slope-destabilising subbundle of a vector bundle $E \rightarrow X$ the $\mathbb{P}(F) \subset \mathbb{P}(E)$ destabilises for suitable polarisations $\pi^{*} L^{m} \otimes \mathcal{O}_{\mathbb{P}(E)}(1), m \gg 0$, on $\mathbb{P}(E)$. Conversely [Ho], if $E$ is slopestable and the base $X$ is cscK with discrete automorphism group then $\mathbb{P}(E)$ is cscK for $m \gg 0$.

- When the base is a curve, we can do better [RT2]. In fact, for $\mathbb{P}(E)$ with discrete automorphism group and any polarisation,

$$
\mathbb{P}(E) \operatorname{cscK} \Longleftrightarrow E \text { HYM } \Longleftrightarrow E \text { stable. }
$$

The converses follow from the Narasimhan-Seshadri theorem [BdB] that stable bundles admit projectively flat connections.

- -1-curves destabilise del Pezzo surfaces $(X, L)$ for appropriate $L$. In particular one can find examples with reductive (even trivial) automorphism group, showing that the folklore conjecture Aut $(X)$ reductive $\Rightarrow \operatorname{cscK}$ does not hold for surfaces. (Tian showed that it does not hold for threefolds [Ti4], but that it does hold for anticanonically polarised surfaces [Ti2].)

- For instance $\mathbb{P}^{2}$ blown up in one point cannot admit a cscK metric for any polarisation because Aut $(X)$ is not reductive. From the above point of view this is because it is destabilised by the exceptional -1-curve for all polarisations.

- Generically stable varieties can specialise to unstable ones. For instance moving two -1-curves together on a stable del Pezzo gives a limiting -2-curve (if the -1-curves arise from blowing up distinct points, then blow up two "infinitely near" points - one point and then another on the exceptional divisor) which can destabilise for suitable $L$.

- Calabi-Yau manifolds, and varieties with canonical singularities and numerically trivial canonical bundle $\left(m K_{X} \sim 0\right)$ are slope stable.

- Canonically polarised varieties with canonical singularities (i.e., the canonical models of Mori theory) are slope stable.

- Remarkably, the product of a (nongeneric) smooth curve with itself can admit polarisations which are slope unstable, giving surfaces of general type which are neither K- nor Chow stable, and which do not admit cscK metrics in that class [Ro].

We will sketch the algebro-geometric proof of the above results later. But differential-geometrically, what the proofs amount to is the following.

We know that the Mabuchi energy (or log-norm functional, in GIT language) is convex over the space of all Kähler metrics on our fixed complex manifold $X$. Intuitively, if it is proper in some sense (roughly, tends to $+\infty$ at infinity) then it should have a unique absolute minimum, which, modulo regularity issues, would be cscK. Conversely the manifold is strictly $\mathrm{K}$-unstable, with no cscK metric, if the Mabuchi energy is unbounded below. If $X$ is slope destabilised by some subscheme $Z$ then there is a family of 
Kähler metrics on $X$, given by "stretching the neck" around $Z$, along which the Mabuchi energy tends to $-\infty$, so $X$ cannot be cscK.

Slope for Chow stability. Fix $Z \subset\left(X, \mathcal{O}_{X}(1)\right) \subset\left(\mathbb{P}^{N}, \mathcal{O}(1)\right)$ embedded by sections of $\mathcal{O}_{X}(1)$, and, as before,

$$
\begin{gathered}
h^{0}\left(\mathcal{O}_{X}(r)\right)=a_{0} r^{n}+a_{1} r^{n-1}+\ldots, \\
h^{0}\left(\mathscr{I}_{Z}^{x r}(r)\right)=a_{0}(x) r^{n}+a_{1}(x) r^{n-1}+\ldots .
\end{gathered}
$$

Then, for all $c \leq \epsilon(Z) \in \mathbb{N}$, define the Chow slope of $Z$ to be

$$
C h_{c}\left(\mathscr{I}_{Z}\right)=\frac{\sum_{i=1}^{c} h^{0}\left(\mathscr{I}_{Z}^{i}(1)\right)}{\int_{0}^{c} a_{0}(x) d x},
$$

a discrete version of (7.1). $Z=\emptyset$ gives

$$
C h(X)=\frac{h^{0}\left(\mathcal{O}_{X}(1)\right)}{a_{0}}=\frac{N+1}{a_{0}},
$$

and we have the following $[\mathbf{R T} 1]$.

Theorem 7.5. Chow (semi)stable $\Rightarrow$ slope (semi)stable:

$$
C h_{c}\left(\mathscr{I}_{Z}\right) \underset{(\leq)}{<} C h(X) \quad \forall Z \subset X .
$$

To see where these results come from, and to explain how far one can get towards a converse, we need to analyse the Hilbert-Mumford criterion for varieties $(X, L)$. We first warm up with a brief overview of the bundle case.

The Hilbert-Mumford criterion for vector bundles. Given a coherent sheaf $E$ on $X$, recall (6.4) how an picking an identification $H^{0}(E(r)) \cong$ $\mathbb{C}^{N}$ for $r \gg 0, N=\chi(E(r))$, realises $E(r)$ as a point of a Quot scheme of quotients

$$
\mathcal{O}(-r)^{\oplus N} \rightarrow E \rightarrow 0 .
$$

Dividing out by the identification, i.e., quotienting the relevant subset of Quot by $S L(N, \mathbb{C})$, gives a moduli space of sheaves.

To apply the Hilbert-Mumford criterion, we consider the $E$-orbit of a 1-PS $\mathbb{C}^{*}<G L(N, \mathbb{C})$ on Quot $[\mathbf{H L}]$ (we shall normalise to $S L(N, \mathbb{C})$ later), whose eigenvalues we can assume are all positive, without loss of generality. The eigenspaces $V_{\lambda}<\mathbb{C}^{N} \cong H^{0}(E(r))$ give a weight filtration $V_{\leq i}=\oplus_{\lambda \leq i} V_{\lambda}$ of $\mathbb{C}^{N}$. Their images $F_{i} \leq E$ under the map (7.6) give a filtration of $E$,

$$
F_{0} \leq F_{1} \leq \ldots \leq F_{p} \leq E,
$$

and the orbit gives a sheaf over $X \times \mathbb{C}$ described in terms of the $F_{i}$ as follows. Let $\mathbb{F}_{i}, \mathbb{E}$ denote the pullbacks of the sheaves $F_{i}, E$ to $X \times \mathbb{C}$, so $\mathbb{F}_{i}=F_{i} \otimes \mathbb{C}[t]$ where $t$ is the variable pulled back from $\mathbb{C}$. Then the orbit gives the following subsheaf of $\mathbb{E}$,

$$
\mathbb{F}_{0}+t \cdot \mathbb{F}_{1}+t^{2} \cdot \mathbb{F}_{2}+\ldots+t^{p} \cdot \mathbb{F}_{p}+t^{p+1} \cdot \mathbb{E}<E \otimes \mathbb{C}[t] .
$$


This is a degeneration of the general fibre $E$ to

$$
F_{0} \oplus F_{1} / F_{0} \oplus \ldots \oplus F_{p} / F_{p-1} \oplus E / F_{p}
$$

over the central fibre $X \times\{0\}$. One can prove this inductively as follows.

Set $Q_{i}=E / F_{i}$, giving exact sequences $0 \rightarrow F_{i} / F_{i-1} \rightarrow Q_{i-1} \rightarrow Q_{i} \rightarrow 0$. In the $p=0$ case, $\mathbb{E}_{0}:=\mathbb{F}_{0}+t . \mathbb{E}$ is the kernel of the composition $\mathbb{E} \rightarrow$ $E \rightarrow Q_{0}$, where the latter two sheaves are considered to be supported on the central fibre $X \times\{0\}$. (So $\mathbb{E}_{0}$ is the elementary transform of $\mathbb{E}$ in $Q_{0}$ on $X \times\{0\}$.) $\mathbb{E}_{0}$ has a map to $F_{0}$ induced by the diagram

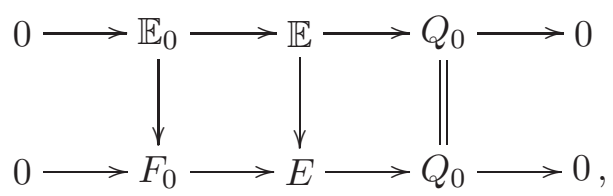

and one to $Q_{0}$ :

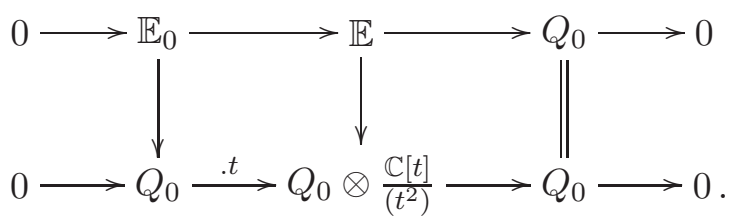

The pair make the central fibre of $\mathbb{E}_{0}$ isomorphic to $F_{0} \oplus Q_{0}$, while the map $\mathbb{E}_{0} \rightarrow Q_{0}$ can be composed with the surjection $Q_{0} \rightarrow Q_{1}$ to continue the induction by defining $\mathbb{E}_{1}$ as its kernel. This is clearly just $\mathbb{F}_{0}+t . \mathbb{F}_{1}+t^{2} \cdot \mathbb{E}$, and similar working shows it has central fibre $F_{0} \oplus F_{1} / F_{0} \oplus Q_{1}$, and so on.

Different 1-PSs can give the same filtration (if the $i$ th piece of the weight filtration of $\mathbb{C}^{N}$ generates the same subsheaf $F_{i} \leq E$ ). But for every filtration $F_{i}$ and sequence of weights there is a canonical least stable 1-PS, given by choosing the weight filtration to be $V_{\leq i}=H^{0}\left(F_{i}(r)\right)<H^{0}(E(r))$.

So we need only consider these canonical 1-PSs: they have the largest GIT weight in their class. Since (7.8) gives the weight space decomposition of the limiting sheaf over the central fibre, the weights of these 1-PSs $\sum_{n} n h^{0}\left(\left(F_{n} / F_{n-1}\right)(k)\right), k \gg 0$, are positive linear combinations of weights of the canonical 1-PSs associated to the splittings

$$
F_{i} \oplus E / F_{i} .
$$

So in fact we need only control the weights of these simpler splittings. Calculating their weights (and then normalising into $S L(N, \mathbb{C})[\mathbf{H L}]$ ) gives the Gieseker stability condition for bundles described earlier, controlled by single subsheaves $F<E$ and their reduced Hilbert polynomials.

The Hilbert-Mumford criterion for varieties. We can now try to do the analogous thing for varieties, following [Mu, RT1].

Any test configuration $(\mathscr{X}, \mathcal{L})$ is $\mathbb{C}^{*}$-birational to $(X \times \mathbb{C}, L)$, so is (a contraction $p$ of) the blow up of $X \times \mathbb{C}$ in a $\mathbb{C}^{*}$-invariant ideal $I$ supported on 
the central fibre, with polarisation $p^{*} \mathcal{L}=\left(\pi^{*} L\right)(-c P)=\pi^{*}\left(L \otimes I^{c}\right)$, where $P$ is the exceptional divisor.

$$
X \times \mathbb{C}^{\pi / \mathrm{Bl}_{I}(X \times \mathbb{C})} \searrow^{p}
$$

Classifying such $\mathbb{C}^{*}$-invariant ideals, there exist subschemes

$$
Z_{p-1} \subseteq \ldots \subseteq Z_{1} \subseteq Z_{0} \subseteq X
$$

with ideal sheaves $\mathscr{I}_{p-1} \supseteq \ldots \supseteq \mathscr{I}_{1} \supseteq \mathscr{I}_{0}$ such that $[\mathbf{M u}]$

$$
I=\mathscr{I}_{0}+t \mathscr{I}_{1}+t^{2} \mathscr{I}_{2}+\ldots+t^{p-1} \mathscr{I}_{p-1}+t^{p} .
$$

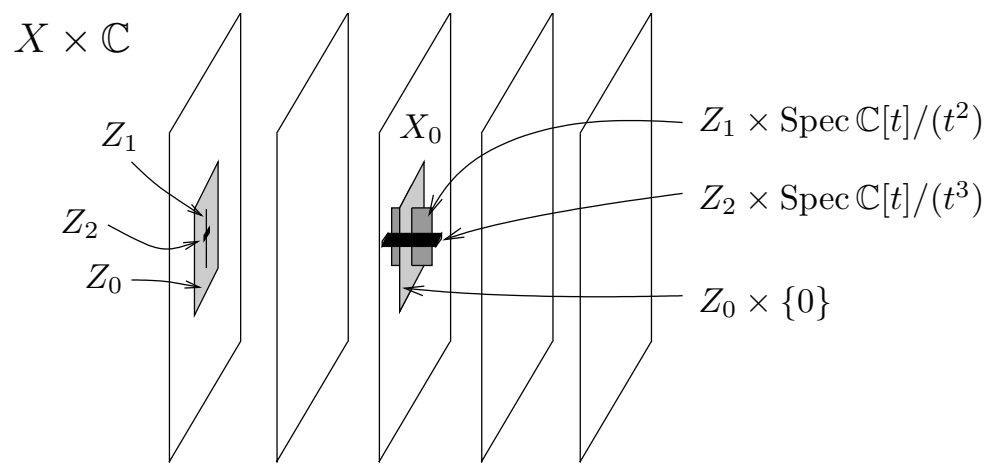

Firstly we have an analogue of the fact that, in the bundle case, one need only consider canonical 1-PSs. Namely, under any map of test configurations like $p$ above, the weights are less stable (more positive) on the dominating test configuration. (Notice that the blow up map $\mathrm{Bl}_{I}(X \times \mathbb{C}) \rightarrow X \times \mathbb{C}$ above is not such a map of test configurations, since it does not preserve polarisations: the line bundle is not a pullback from downstairs.)

Proposition 7.14. Suppose $X$ is normal. Given a test configuration $(\mathscr{X}, \mathcal{L})$ for $(X, L)$ and another flat $\mathbb{C}^{\times}$-family $\mathscr{Y} \rightarrow \mathbb{C}$ with a birational $\mathbb{C}^{*}$-equivariant map $p: \mathscr{Y} \rightarrow \mathscr{X}$, there exists an a $\geq 0$ such that

$$
w\left(H_{\mathscr{X}_{0}}^{0}\left(\mathcal{L}^{k}\right)\right)=w\left(H_{\mathscr{Y}}^{0}\left(p^{*} \mathcal{L}^{k}\right) / t H_{\mathscr{Y}}^{0}\left(p^{*} \mathcal{L}^{k}\right)\right)-a k^{n}+O\left(k^{n-1}\right) .
$$

(Here $w$ denotes the total weight of a $\mathbb{C}^{*}$-action - i.e. its weight on the determinant of the $\mathbb{C}^{*}$-module. The normality of $X$ is required to equate $H^{0}\left(X, L^{k}\right)$ with sections of $p^{*} \mathcal{L}^{k}$ on a general fibre of $\mathscr{Y}$. The result is stated in rather more generality than we require here (in particular allowing $\mathscr{Y}$ to have general fibre some blow up of $X$, rather than just $X$ ) for future use. We are forced to use $H_{\mathscr{Y}}^{0}\left(p^{*} \mathcal{L}^{k}\right) / t H_{\mathscr{Y}}^{0}\left(p^{*} \mathcal{L}^{k}\right)$, rather than $H_{\mathscr{Y}_{0}}^{0}\left(p^{*} \mathcal{L}^{k}\right)$, because $p^{*} \mathcal{L}$ need not be ample on $\mathscr{Y}$.)

So we need only consider weights on the normalisation of the blow up of $X \times \mathbb{C}$ in $I$, as this is is itself a perfectly good test configuration. (In the Chow stability case this test configuration may not arise from a linear 
transformation of the given projective space - only from an embedding by a higher twist of $L$ - but this does not concern us as Proposition 7.14 gives an equality in weights to $O\left(k^{n}\right)$, which is all that is required for Chow stability.)

Next we consider the easiest case of $p=1$, i.e., $I=\mathscr{I}_{0}+(t)$. So we blow up in $Z_{0} \times\{0\} \subset X \times \mathbb{C}$, giving the deformation to normal cone of $Z_{0}$. This is the analogue of the simplest degenerations of bundles and sheaves earlier; the canonical degenerations of a one-step filtration to a direct sum (7.10).

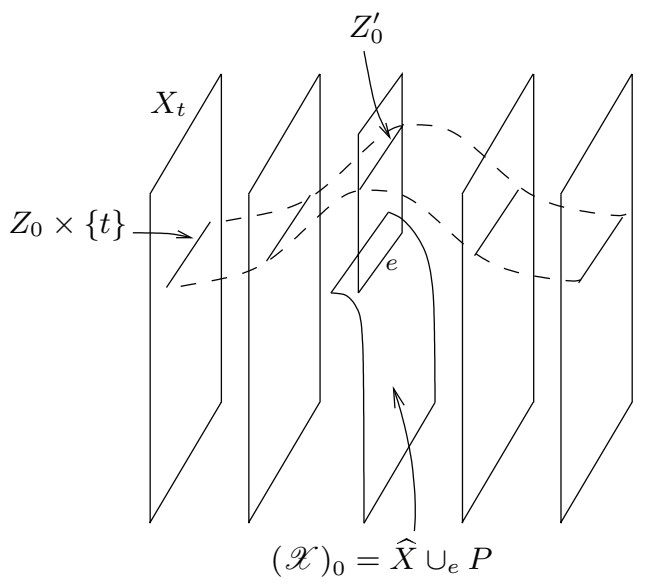

The exceptional divisor $P$ is the normal cone of $Z_{0}$ : if $Z_{0} \subset X$ is smooth then this is the projective completion $\mathbb{P}\left(\nu_{Z_{0}} \oplus \underline{\mathbb{C}}\right) \rightarrow Z_{0}$ of the normal bundle $\nu_{Z_{0}} \rightarrow Z_{0}$.

$\mathbb{C}^{*} \ni \lambda$ acts on the blow up (as $[1: \lambda]=\left[\lambda^{-1}: 1\right]$ on $\mathbb{P}\left(\nu_{Z_{0}} \oplus \underline{\mathbb{C}}\right)$ in the smooth case) and on the line bundle $\pi^{*} L(-c P)$ over $\mathrm{Bl}_{Z_{0} \times\{0\}}(X \times \mathbb{C})$.

This deformation to the normal cone of $Z$ replaces $H_{X}^{0}\left(L^{r}\right)$ (filtered by $\left.H^{0}\left(L^{r} \otimes \mathscr{I}_{Z}^{j}\right)\right)$ by the associated graded of the filtration on the central fibre:

$$
\begin{aligned}
H_{X}^{0}\left(\mathscr{I}_{Z}^{c r}(r)\right) \oplus t H_{X}^{0}\left(\mathscr{I}_{Z}^{c r-1}(r) / \mathscr{I}_{Z}^{c r}(r)\right) \oplus \ldots \\
\oplus t^{c r-1} H_{X}^{0}\left(\mathscr{I}_{Z}(r) / \mathscr{I}_{Z}^{2}(r)\right) \oplus t^{c r} H_{X}^{0}\left(\mathcal{O}_{Z}(r)\right) .
\end{aligned}
$$

Here $t$ is the coordinate on the $\mathbb{C}$-base. This is the splitting of sections of $L^{r}(-c r P)$ on the central fibre into those on the proper transform of the original central fibre $X$ (the first term) plus the polynomials on $P$. In turn the latter can be split into their $\mathbb{C}^{*}$-weight spaces as $t^{j}$ times by the homogeneous polynomials on the normal bundle of $Z \subset X$ of degree $c r-j$.

So this is the weight space decomposition, with $\mathbb{C}^{*}$ acting on $t^{j}$ with weight $-j$, and the weight on top exterior power is

$$
\begin{aligned}
w_{r} & =-\sum_{j=1}^{c r} j h_{X}^{0}\left(\mathscr{I}_{Z}^{c r-j}(r) / \mathscr{I}_{Z}^{c r-j+1}(r)\right) \\
& =-\sum_{j=1}^{c r} h^{0}\left(\mathscr{I}_{Z}^{j}(r)\right)-\operatorname{crh}^{0}\left(\mathcal{O}_{X}(r)\right) .
\end{aligned}
$$


This looks like a discrete approximation (Riemann sum) for

$$
\int_{0}^{c} h^{0}\left(\mathscr{I}_{Z}^{x r}(r)\right) d x
$$

so we estimate it by the trapezium rule, giving

$$
-\left(\int_{0}^{c} a_{0}(x) d x\right) r^{n+1}+\int_{0}^{c}\left(a_{1}(x)+\frac{a_{0}^{\prime}(x)}{2}\right) d x r^{n}+O\left(r^{n-1}\right) .
$$

Normalising (to make the 1-PS lie in $S L(N, \mathbb{C})$ instead of $G L(N, \mathbb{C})$ ) we find the K-stability slope criterion of (7.1) and Theorems 7.2 and 7.3.

Alternatively, taking the leading order term of (7.15) and normalising gives the Chow slope criterion of Theorem 7.5.

General test configurations. So we have the analogue of the result in the bundle case that one need only consider canonical 1-PS orbits - i.e. we need only consider test configurations that are normalisations of the form (7.11). And we have the analogue of the simple degenerations (7.10), given by the deformation to the normal cone of a subscheme $Z \subset X$ yielding the right analogue of slope stability. For bundles any canonical 1-PS weight turned out to be a positive linear combination of these simple weights, so ideally one would like to write the weight of a test configuration (7.11) as a positive linear combination of weights (7.15) - if so one could conclude that stability of varieties was equivalent to slope stability.

So we need the analogue of the induction (7.9) that we did in the bundle case to handle (7.7). In fact it turns out we can mirror it almost completely; moreover the bundle induction is a special case of what follows below when we consider the sheaf $\mathbb{E}$ of (7.7) to be the sections of the polarisation $\mathcal{O}_{\mathbb{P}\left(E^{*}\right)}(1)$ on the variety $\mathbb{P}\left(E^{*}\right) \times \mathbb{C}$. The correspondence between (7.7) and (7.13) is clear, and the elementary transformations we did in the bundle case become, on projectivisation, the blowups below.

The proper transform $\overline{Z_{0} \times \mathbb{C}}$ of $Z_{0} \times \mathbb{C}$ is flat over the base $\mathbb{C}$. It defines a copy $Z_{0}^{\prime}$ of $Z_{0}$ in the central fibre of the deformation to the normal cone of $Z_{0}$ : 


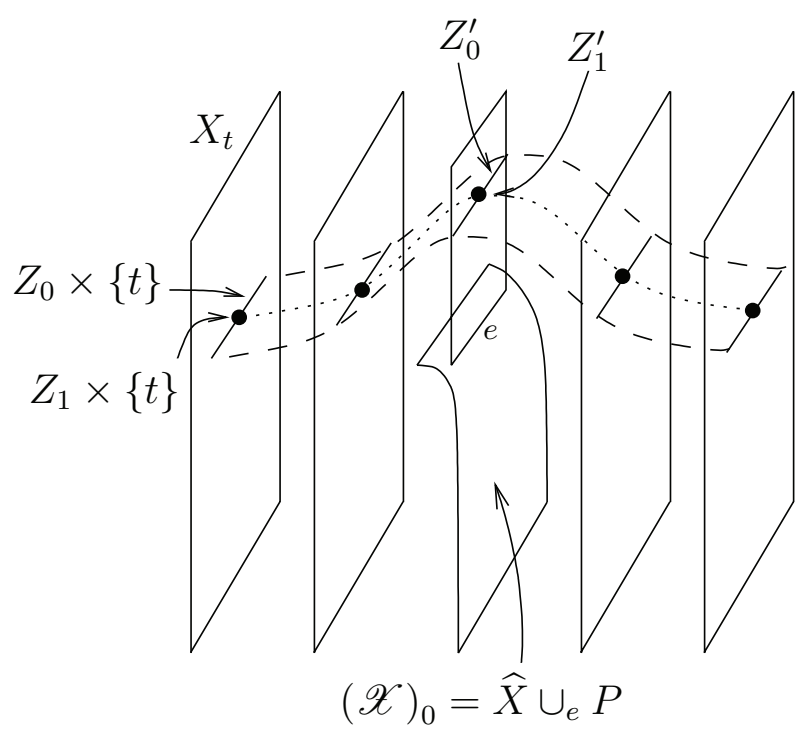

By (7.12) this defines subschemes $Z_{p-1}^{\prime} \subseteq \ldots \subseteq Z_{1}^{\prime} \subset Z_{0}^{\prime}$. So next we blow up in $Z_{1}^{\prime}$, giving $Z_{p-1}^{\prime \prime} \subseteq \ldots \subseteq Z_{1}^{\prime \prime}$; next blow up $Z_{2}^{\prime \prime}$, and so on inductively up to $Z_{p-1}^{(p-1)}$.

(For $X=\mathbb{P}\left(E^{*}\right)$ and the degeneration (7.7), we are blowing up the subschemes $\mathbb{P}\left(Q_{0}^{*}\right)$ (in the central fibre), then the central fibre of the proper transform of $\mathbb{P}\left(Q_{1}^{*}\right)$, and so on; but this is just the projectivisation of the elementary transformations (7.9).)

Theorem 7.16. The blow up of $X \times \mathbb{C}$ in $I=\mathscr{I}_{0}+t \mathscr{I}_{1}+\ldots+t^{p-1} \mathscr{I}_{p-1}+$ $t^{p}$ is a contraction of this iterated blow up.

This is meant in the $\mathbb{C}^{*}$-equivariant polarised sense - the polarisation $L(-c P)$ on $\mathrm{Bl}_{I}(X \times \mathbb{C})$ is the pullback of the natural polarisation on the iterated blow up given by starting with $L$ and, at each stage, pulling back and subtracting $c$ times the exceptional divisor.

Thus, by our result (Proposition 7.14) that one need only calculate weights on dominating test configurations, we are left with calculating the amount that each blow up in $Z_{i}^{(i)}$ adds to the weight of the resulting $\mathbb{C}^{*}$ action on the determinant of the space of sections of the $r$ th power of the polarisation on the central fibre.

THEOREM 7.17. Consider the ith step, when we blow up $Z_{i}^{(i)}$. If all thickenings of $\overline{\left(Z_{i} \times \mathbb{C}\right)}$ are flat over $\mathbb{C}$ then this adds $w\left(Z_{i}\right)$ to the weight, to $O\left(r^{n}\right)$.

(Here $w\left(Z_{i}\right)$ is weight on deformation to normal cone of $Z_{i}$.)

In fact under certain conditions one can get the result to $O\left(r^{n-1}\right)$ [RT1].

So if this flatness condition holds, the total (normalised) weight is $w\left(Z_{0}\right)$ $+\cdots+w\left(Z_{p-1}\right)$. So $X$ is stable if and only if

$$
w\left(Z_{0}\right)+\ldots+w\left(Z_{p-1}\right) \prec 0 \Longleftrightarrow w(Z) \prec 0 \forall Z .
$$


So if this flatness condition held in general, then stability and slope stability would be equivalent.

The flatness problem. In fact $\overline{\left(Z_{i} \times \mathbb{C}\right)}$ is flat over $\mathbb{C}$, but since blow ups use all powers of an ideal, we require all of its scheme theoretic thickenings $k \overline{\left(Z_{i} \times \mathbb{C}\right)}$ (defined by the ideal $\mathscr{I} \frac{k}{Z_{i} \times \mathbb{C}}$ ) to be flat too. The idea of the proof of Theorem 7.17 is then that a formal neighbourhood of $Z_{i}^{(i)}$ in the test configuration looks sufficiently like a formal neighbourhood of $Z_{i} \times\{0\} \subset X \times \mathbb{C}$ for the weights added by the two blow ups to be comparable to $O\left(r^{n}\right)$ - the corrections being due to estimates on the sizes of nonvanishing cohomology groups.

This flatness condition holds for $Z_{i} \subset X$ smooth, or reduced simple normal crossing (snc) divisors.

In general, one can use resolution of singularities

$$
\left(X \supset Z_{i}\right) \stackrel{\pi}{\longleftarrow}\left(\widehat{X} \supset m_{i} D_{i}\right), \quad D_{i} \text { snc divisors, }
$$

to replace $(X, L)$ by $\left(\widehat{X}, \pi^{*} L\right)$. Test configurations for the latter dominate those of the former, so Proposition 7.14 allows us to obtain (7.18) for $X$ normal, so long as $m_{i}=1$ for all $i$.

So finally we need to be able to deal with the snc divisors $D_{i}$ being possibly nonreduced. We can attempt to do this by basechange [RT1], which we illustrate with an example.

Consider the case where $Z_{0} \subset X$ is a double point in a smooth curve. Locally then $\mathscr{I}_{0}=\left(x^{2}\right)$, and the deformation to normal cone of $Z_{0}$ is the blow up of $X \times \mathbb{C}$ in $\left(x^{2}, t\right)$.

Now consider squaring the $\mathbb{C}^{*}$-action. This is trivial from a GIT point of view (it just doubles the weight, which we can later halve). But it fundamentally alters our geometric description of the test configuration, blowing up $X \times \mathbb{C}$ in the ideal $\left(x^{2}, t^{2}\right)$.

Taking the integral closure of this ideal corresponds to normalising the blow up, which we can deal with by Proposition 7.14. That is, we get a more unstable test configuration by blowing up in $\left(x^{2}, x t, t^{2}\right)=(x, t)^{2}$, and it suffices to control the weights of this test configuration.

But this is now a much nicer ideal, and corresponds to blowing up in $(x, t)$ and using a different line bundle (squaring the exceptional divisor $P \mapsto 2 P$ or $c \mapsto 2 c$ ). So modulo doubling $c$ and the weight, we have removed the multiplicity 2 of the double point $Z_{0}$.

In this way we can deal with $D_{i}$ with multiplicities $m_{i}$ when they all have the same support. This is enough to prove that (K- and Chow) stability coincides with (K- and Chow) slope stability for smooth curves, and indeed gives probably the "right" geometric proof, rather than the old combinatorial (for Chow stability) and analytical (for K-stability) proofs.

Of course, for higher dimensions, one would like to combine the two approaches to deal with snc divisors with intersecting components of different multiplicities, for instance, $D_{0}=\left(x^{2} y=0\right), D_{1}=(x=0)$. This is still 
work in progress, but its difficulty suggests that slope stability is not enough to describe stability (unlike for the more linear bundle case) except in one dimension or on projective bundles over stable bases.

\section{References}

[AB] M.F. Atiyah and R. Bott, The Yang-Mills equations over Riemann surfaces, Phil. Trans. R. Soc. Lond. Ser. A 308 (1982), 523-615.

[ACGT] V. Apostolov, D. Calderbank, P. Gauduchon, and W. Tønnesen-Friedman, Hamiltonian 2-forms in Kähler geometry, III Extremal metrics and stability, math.DG/0511118, 2005.

[AP] C. Arezzo and F. Pacard, Blowing up Kähler manifolds with constant scalar curvature, II, math.DG/0504115, 2004.

[Au] T. Aubin, Équations du type Monge-Ampère sur les variétés kähleriennes compactes, C.R. Acad. Sci. Paris Sér. A-B 283 (1976), A119-A121.

[BM] S. Bando and T. Mabuchi, Uniqueness of Einstein-Kähler metrics modulo connected group actions, in 'Algebraic Geometry', Advanced Studies in Pure Math. 10 (1987), 11-40.

[Bi] O. Biquard, Métriques kählériennes à courbure scalaire constante, Séminaire Bourbaki 938, 2004.

[Bo] J.-P. Bourguignon, Métriques d'Einstein-Kähler sur les variétés de Fano: obstructions et existence (d'après Y. Matsushima, A. Futaki, S.-T. Yau, A. Nadel et G. Tian), Séminaire Bourbaki, Astérisque 245 (1997), 277-305.

[BdB] D. Burns and P. De Bartolomeis, Stability of vector bundles and extremal metrics, Invent. Math. 92 (1988), 403-407.

[CGR] M. Cahen, S. Gutt, and J. Rawnsley, Quantization of Kähler manifolds, II, Trans. Amer. Math. Soc. 337 (1993), 73-98.

[Ca] E. Calabi, Extremal Kähler metrics, in 'Seminar on differential geometry' (S.-T. Yau, ed.), Princeton Univ. Press, New Jersey, 259-290, 1982.

[Ch] X. Chen, The space of Kähler metrics, Jour. Differential Geom. 56 (2000), 189234.

[CT] X. Chen and G. Tian, Geometry of Kähler metrics and holomorphic foliation by discs, math.DG/0409433, 2004.

[DT] W. Ding and G. Tian, Kähler-Einstein metrics and the generalized Futaki invariant, Invent. Math. 110 (1992), 315-335.

[Dl] I. Dolgachev, Lectures on invariant theory, LMS Lecture Note Series, 296, Cambridge University Press, 2003.

[DH] I. Dolgachev and Y. Hu, Variation of Geometric Invariant Theory Quotients, Publ. IHES 87 (1998), 5-56.

[Do1] S.K. Donaldson, Remarks on gauge theory, complex geometry and 4-manifold topology, in 'Fields Medallists Lectures', World Sci. Ser. 20th Century Math., 5, World Sci. Publishing, River Edge, NJ, 384-403, 1997.

[Do2] S.K. Donaldson, Geometry in Oxford c. 1980-85, Sir Michael Atiyah: a great mathematician of the twentieth century, Asian J. Math. 3 (1999), xliii-xlvii.

[Do3] S.K. Donaldson, Scalar curvature and projective embeddings, I, Jour. Differential Geom. 59 (2001), 479-522.

[Do4] S.K. Donaldson, Planck's constant in complex and almost-complex geometry, XIIIth International Congress on Mathematical Physics (London, 2000), 63-72, Int. Press, Boston, 2001.

[Do5] S.K. Donaldson, Scalar curvature and stability of toric varieties, Jour. Differential Geom. 62 (2002), 289-349. 
[Do6] S.K. Donaldson, Lower bounds on the Calabi functional, math.DG/0506501, 2005.

[DK] S.K. Donaldson and P. Kronheimer, The geometry of four-manifolds, Oxford Univ. Press, 1990.

[Fi] J. Fine, Constant scalar curvature Kähler metrics on fibred complex surfaces, Jour. Differential Geom. 68 (2004), 397-432.

[Fj] A. Fujiki, Moduli space of polarized algebraic manifolds and Kähler metrics, Sugaku 42 (1990), 231-243; English translation: Sugaku Expo. 5 (1992), 173-191.

[Fu] A Futaki, An obstruction to the existence of Einstein-Kähler metrics, Invent. Math. 73 (1983), 437-443.

[Ho] Y-J. Hong, Gauge-fixing constant scalar curvature equations on ruled manifolds and the Futaki invariants, Jour. Differential Geom. 60 (2002), 389-453.

[HL] D. Huybrechts and M. Lehn, Geometry of moduli spaces of shaves, Aspects in Mathematics, Vol. E31, Vieweg, 1997.

[Hu] Y. Hu, Topological Aspects of Chow Quotients, math.AG/0308027.

[Ki] F.C. Kirwan, Cohomology of quotients in symplectic and algebraic geometry, Mathematical Notes, 34, Princeton University Press, Princeton, 1984.

[LB] C. LeBrun, Anti-self-dual metrics and Kähler geometry, Proceedings of the International Congress of Mathematicians (Zürich, 1994), 498-507, Birkhäuser, 1995.

[Mb1] T. Mabuchi, K-energy maps integrating Futaki invariants, Tohoku Math. Jour. 38 (1986), 245-257.

[Mb2] T. Mabuchi, An energy-theoretic approach to the Hitchin-Kobayashi correspondence for manifolds, I, Invent. Math. 159 (2004), 225-243.

[Mu D. Mumford, Stability of projective varieties, Enseignement Math. (2) 23 (1977), 39-110.

[MW] W. Müller and K. Wendland, Extremal Kähler metrics and Ray-Singer analytic torsion, in 'Geometric aspects of partial differential equations' (Roskilde, 1998), 135-160, Contemp. Math., 242, Amer. Math. Soc., Providence, RI, 1999.

[GIT] D. Mumford, J. Fogarty, and F. Kirwan, Geometric Invariant Theory, Third edition, Erg. Math., 34, Springer-Verlag, Berlin, 1994.

[Na] A. Nadel, Multiplier ideal sheaves and Kähler-Einstein metrics of positive scalar curvature, Ann. of Math. 132 (1990), 549-596.

[Ne] P. Newstead, Introduction to moduli problems and orbit spaces, Tata Institute, Bombay. Springer-Verlag, 1978, out of print.

[RS] Y. Rollin and M. Singer, Non-minimal scalar-flat Kähler surfaces and parabolic stability, Invent. Math. 162 (2005), 235-270.

[Ro] J. Ross, Unstable products of smooth curves, to appear in Invent. Math., math.AG/0506447, 2005.

[RT1] J. Ross and R. Thomas, A study of the Hilbert-Mumford criterion for the stability of projective varieties, math.AG/0412519, 2004.

[RT2] J. Ross and R. Thomas, An obstruction to the existence of constant scalar curvature Kähler metrics, to appear in Jour. Differential Geom., math.DG/0412518, 2004.

[S] Y.T. Siu, The existence of Kähler-Einstein metrics on manifolds with positive anticanonical line bundle and a suitable finite symmetry group, Ann. of Math. 127 (1988), 585-627.

[Sz1] G. Székelyhidi, Extremal metrics and K-stability, math.AG/0410401, 2004.

[Sz2] G. Székelyhidi, Ph.D. thesis, Imperial College London, 2006, in preparation.

[Th] M. Thaddeus, Geometric invariant theory and flips, J. Amer. Math. Soc. 9 (1996), 691-723.

[Ti1] G. Tian, On Kähler-Einstein metrics on certain Kähler manifolds with $C_{1}(M)>$ 0, Invent. Math. 89 (1987), 225-246. 
[Ti2] G. Tian, On Calabi's conjecture for complex surfaces with positive first Chern class, Invent. Math. 101 (1990), 101-172.

[Ti3] G. Tian, On a set of polarized Kähler metrics on algebraic manifolds, Jour. Differential Geom. 32 (1990), 99-130.

[Ti4] G. Tian, Kähler-Einstein metrics with positive scalar curvature, Invent. Math. 130 (1997), 1-37.

[Wa1] X.-W. Wang, Balance point and Stability of Vector Bundles Over a Projective Manifold, Math. Res. Lett. 9 (2002), 393-411.

[Wa2] X.-W. Wang, Canonical metrics on stable vector bundles, Comm. Anal. Geom. 13 (2005), 253-285.

[Y1] S.-T. Yau, On the Ricci curvature of a compact Kähler manifold and the complex Monge-Ampère equation, I, Comm. Pure Appl. Math. 31 (1978), 339-411.

[Y2] S.-T. Yau, Nonlinear analysis in geometry, Enseign. Math. 33 (1987), 109-158.

[Y3] S.-T. Yau, Open problems in geometry, in 'Differential geometry: partial differential equations on manifolds' (Los Angeles, CA, 1990), 1-28, Proc. Sympos. Pure Math., 54, AMS publications, 1993.

[Zh] S. Zhang, Heights and reductions of semi-stable varieties, Compositio Math. 104 (1996), 77-105. 